\title{
Creating HiRISE digital elevation models for Mars using the open-source Ames Stereo Pipeline
}

\author{
Adam J. Hepburn ${ }^{1,2}$, Tom Holt ${ }^{1}$, Bryn Hubbard ${ }^{1}$, and Felix $\mathbf{~ N g}^{2}$ \\ ${ }^{1}$ Geography and Earth Sciences, Aberystwyth University, Llandinam Building, Penglais Campus, Aberystwyth, \\ SY23 3DB, UK \\ ${ }^{2}$ Department of Geography, The University of Sheffield, Sheffield, S10 2TN, UK
}

Correspondence: Adam J. Hepburn (adh28@aber.ac.uk)

Received: 13 March 2019 - Discussion started: 26 June 2019

Revised: 27 September 2019 - Accepted: 30 September 2019 - Published: 9 December 2019

\begin{abstract}
The present availability of sub-decametre digital elevation models on Mars - crucial for the study of surface processes - is scarce. In contrast to low-resolution global datasets, such models enable the study of landforms $<10 \mathrm{~km}$ in size, which is the primary scale at which geomorphic processes have been active on Mars over the last $10-20 \mathrm{Myr}$. Stereogrammetry is a means of producing digital elevation models from stereo pairs of images. The HiRISE camera on board the Mars Reconnaissance Orbiter has captured $>3000$ stereo pairs at $0.25 \mathrm{~m} \mathrm{pixel}^{-1}$ resolution, enabling the creation of high-resolution digital elevation models $\left(1-2 \mathrm{~m} \mathrm{pixel}^{-1}\right)$. Hitherto, only $\sim 500$ of these pairs have been processed and made publicly available. Existing pipelines for the production of digital elevation models from stereo pairs, however, are built upon commercial software, rely upon sparsely available intermediate data, or are reliant on proprietary algorithms. In this paper, we present and test the output of a new pipeline for producing digital elevation models from HiRISE stereo pairs that is built entirely upon the open-source NASA Ames Stereo Pipeline photogrammetric software, making use of freely available data for cartographic rectification. This pipeline is designed for simple application by researchers interested in the use of high-resolution digital elevation models. Implemented here on a research computing cluster, this pipeline can also be used on consumer-grade UNIX computers. We produce and evaluate four digital elevation models using the pipeline presented here. Each are globally well registered, with accuracy similar to those of digital elevation models produced elsewhere.
\end{abstract}

\section{Introduction}

After several decades of orbital study, Mars is now well served by remotely sensed imagery. This imagery enables the detailed examination and identification of features and processes globally at a spatial resolution of $\sim 6 \mathrm{~m} \mathrm{pixel}^{-1}$, and in many places down to $0.25 \mathrm{~m}_{\text {pixel }}{ }^{-1}$ spatial resolution. However, Mars is poorly served by accurate, high-resolution digital elevation models (DEMs), which are critical resources for the study of surface processes. DEMs enable an understanding of 3-D form and process relationships otherwise only available through in situ study. These include investigations of recurring slope lineations (Schaefer et al., 2019), glacial deposits (Hubbard et al., 2011; Conway and Balme, 2014), aeolian ridges (Berman et al., 2018; Chojnacki et al., 2014, 2017), and fluvial deposits (Voigt and Hamilton, 2018; Tirsch et al., 2018; Adler et al., 2019; Davis et al., 2018; Hamilton et al., 2018; Stack et al., 2016; Wilson et al., 2018). DEMs also fulfil a critical mission requirement when searching for potential rover landing sites (Stack et al., 2016; Golombek et al., 2017, 2012; Grant et al., 2018). Finally, high-resolution DEMs add crucial contextual information to other forms of remote-sensing data. DEMs then are vital in planetary sciences, in which in situ study is limited to only a handful of lander missions. Several martian DEM datasets have been produced to fulfil this requirement, but they vary dramatically in both resolution and coverage.

The Mars Orbiter Laser Altimeter (MOLA) instrument aboard the Mars Global Surveyor (MGS) is the only dedicated altimeter to orbit Mars. It provides a globally complete model of Mars's elevation, with a vertical accuracy of $\sim 1.5 \mathrm{~m}$ (Smith et al., 2001). MOLA has proved extremely 
useful for the study of the largest topographic features on Mars. These features, on the scale of the Tharsis bulge and the dichotomy boundary (1000s of kilometres), exert an influence on the regional-global scale topography of Mars and reflect large changes in Mars's early geological history (Mège and Masson, 1996; Sharp, 1973; Frey et al., 1998). However, with an along track resolution of $300 \mathrm{~m}$ and an interpolated grid spacing of $\sim 460 \mathrm{~m}$ at the Equator (Smith et al., 2001), MOLA has limited potential for the study of features $<10 \mathrm{~km}$ in scale. During the last 2 billion years, substantial dynamic landscape evolution has occurred at or below this scale (Day and Kocurek, 2016; Andrieu et al., 2018; Banks et al., 2018; Aye et al., 2019). These features are increasingly well covered by 2-D imagery, but there is great demand for DEMs with horizontal resolution much better than MOLA's that can be combined with imagery to resolve these landscapes.

Stereogrammetry allows information in the third dimension to be determined from two overlapping 2-D images and thus DEM generation, which should be prolific for many areas on Mars given the abundance of high-resolution imagery. By using the parallax or difference in viewing angles between two images (Fig. 1), stereogrammetry can be used for not only determining the relative topographic change in a landscape but also the elevation in absolute terms (Fenton and Herkenhoff, 2000). In the simplest sense, stereogrammetry is the estimation of 3-D coordinates from the measurement of common points between two 2-D images (whose 2-D coordinates may or may not be known, Beyer et al., 2018). On Earth, this technique has been used to great effect in the Arctic, where a constellation of satellites has returned enough stereo-imagery for widespread DEM coverage at $2 \mathrm{~m} \mathrm{pixel}^{-1}$ resolution across multiple time windows (See ArcticDEM: Noh and Howat, 2017).

The High Resolution Stereo Camera (HRSC) instrument aboard the Mars Express satellite is the only existing purpose-built stereogrammetric camera to orbit Mars. HRSC includes a push-broom stereo sensor that acquires images along track and uses the spacecraft motion to record images of the surface from different perspectives (Jaumann et al., 2007; Gwinner et al., 2016). HRSC has acquired a globally expansive series of stereogrammetric DEMs in this manner, with a best grid-resolution of $\sim 75 \mathrm{~m}$. DEMs can also be made using higher-resolution imagery; HRSC DEMs, while valuable in their own right, also serve as a resolution bridge between the coarse MOLA DEM and DEMs produced using the higher-resolution Mars Reconnaissance Or-

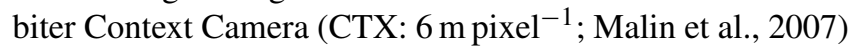
and High Resolution Image Science Experiment (HiRISE: $25-50 \mathrm{~cm} \mathrm{pixel}^{-1}$; Mcewen et al., 2007) imagery.

Herein, we focus on CTX and HiRISE datasets to produce DEMs. The primary science mission of the CTX and HiRISE cameras is not to create DEMs, and as such they are not arranged for the acquisition of complete stereo pairs per orbit. Instead, stereo pairs of images are acquired over separate orbits often months apart, as and when the spacecraft's flight path permits. Nonetheless, $>3000$ stereo pairs of HiRISE imagery and $>1600$ stereo CTX pairs have been captured in this way (Tao et al., 2018). Each of these CTX and HiRISE stereo pairs can be used to create DEMs at resolutions of $24 \mathrm{~m}$ and 1-2 $\mathrm{m}$ respectively (Tao et al., 2018). However, due to the time intensive and manual nature of the DEM processing pipeline used, only $\sim 500$ of these HiRISE pairs have been processed and released on the Planetary Data System Orbital Data Explorer (PDS ODE: Tao et al., 2018). No CTX DEMs have been released (Tao et al., 2018). The current scarcity of HiRISE DEMs at $1-2 \mathrm{~m}$ resolution limits their application to studying landforms at the decametre scale.

Given the increasing volume of stereo pairs that remain unprocessed, and to better enable the utilization of highresolution DEMs that they can be used to create, there is a strong motivation for moving away from the traditionally somewhat esoteric means of producing DEMs towards more open-source and automated methods (Moratto et al., 2010; Beyer et al., 2018). Accordingly, in this paper we present and test an open-source and largely automated processing pipeline for the production of well registered and globally accurate DEMs from stereo pairs of HiRISE and CTX images, referred to hereafter as the Aberystwyth University pipeline (AU pipeline). Notably, our methods presented herein are focussed on the proof of concept and functionality of this new pipeline. The AU pipeline is designed to be rapidly and freely implemented by researchers and is based on the open-source suite of automated geodesy and stereogrammetry tools from the NASA intelligent systems division called the Ames Stereo Pipeline (ASP) (Moratto et al., 2010; Beyer et al., 2018) and the Integrated Software for Imagers and Spectrometers version 3.5.3 programme (ISIS v3.5.3) (Anderson et al., 2004; Anderson, 2008). Several pipelines already exist for the generation of HiRISE and CTX digital elevation models, using both open-source and commercial software. The AU pipeline is designed to be freely implemented, and as such we describe only those pipelines making use of open-source software and refer readers to Tao et al. (2018) for a summary of the existing SOCET-SET pipeline for the production of HiRISE DEMs released on the PDS ODE.

This paper is organized into the following sections: in Sect. 2 we describe the existing open-source pipelines for producing DEMs; first reviewing the ASP method for producing DEMs in Sect. 2.1, which serves as a useful overview of stereogrammetry in general; in Sects. 2.3-2.4 we go on to review the Chicago (Mayer and Kite, 2016) and University College London (UCL) CASP-GO (Tao et al., 2018) pipelines, describing the current state of the art open-source stereogrammetric methods; finally, in Sect. 3 we present our new open-source pipeline for the production of DEMs from stereo image pairs, which is tested thereafter in Sects. 3.2-4. 

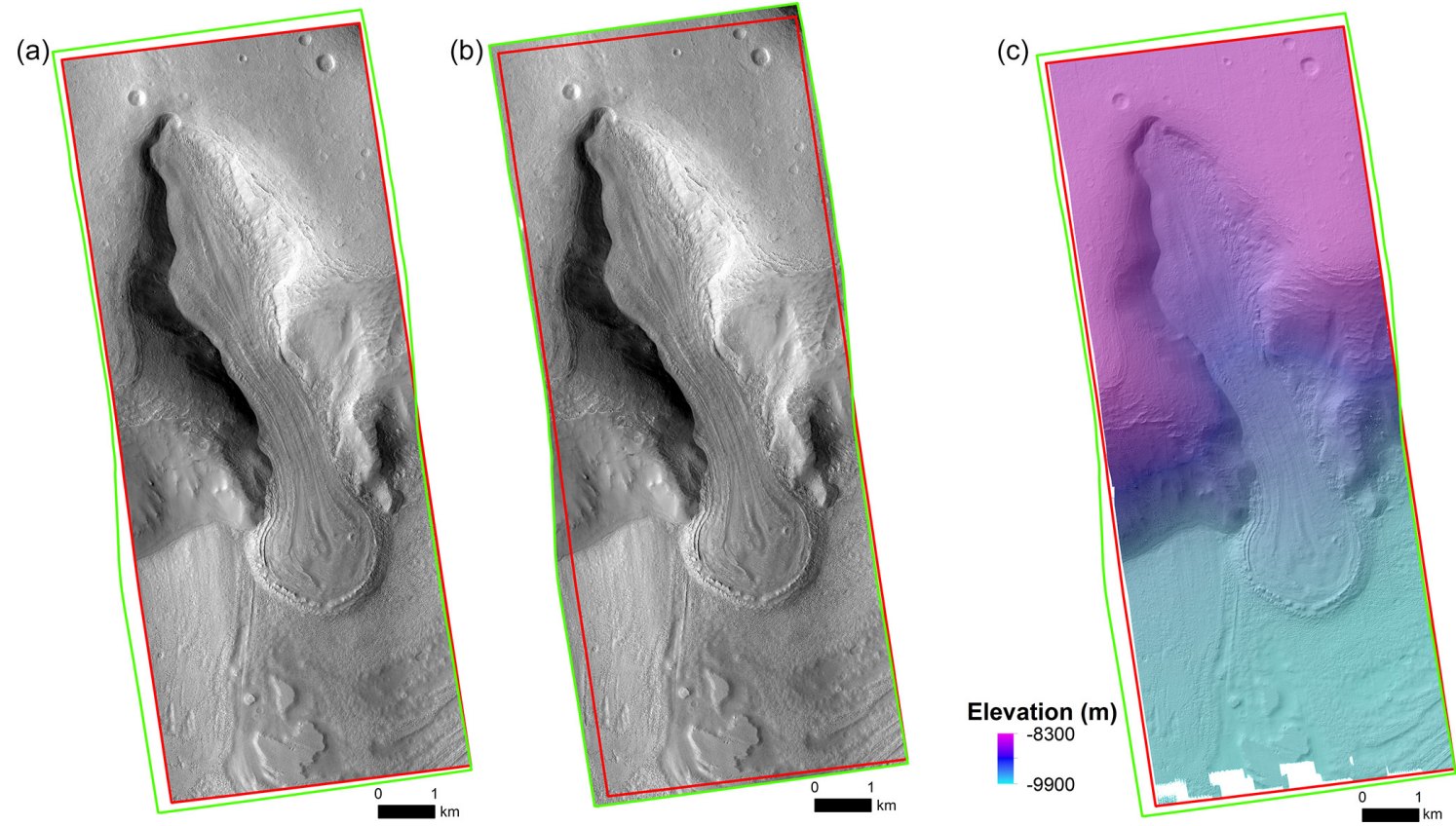

Figure 1. Inputs and outputs of the stereogrammetry process. Here, a left map-projected image (a: HiRISE EDR: ESP_052535_2225) and right map-projected image (b: HiRISE EDR: ESP_052324_2225) are passed to the ASP stereo programme. Stereo uses the camera information associated with the input images to assign 3-D coordinates to each cell. The output point cloud from this process can then be converted to a DEM at 1-2 $\mathrm{mpixel}^{-1}$ resolution (c), and hillshaded to aid interpretation. The glacier-like form visible here, has been a frequent target of HiRISE imagery, but 3-D models enable one to interrogate how this feature may flow downslope. This figure and all subsequent figures are orientated northwards unless it is indicated otherwise.

\section{Existing open-source DEM generation pipelines}

\subsection{The ASP method}

The simplest way to produce HiRISE-CTX DEMs is implemented within the ASP programme (Moratto et al., 2010; Beyer et al., 2018). Written on the vision workbench, a general purpose image processing library built into the United States Geological Survey's (USGS) ISIS programme (Anderson et al., 2004; Anderson, 2008), ASP allows the nearautomatic processing of stereo imagery from NASA rovers and orbiters - and commercial Earth-orbiting platforms - to produce cartographic outputs (Beyer et al., 2018). As input, ASP takes the same proprietary cube (.cub) file format as ISIS. For both CTX and HiRISE images, a series of preprocessing steps are necessary to convert the experimental data record (EDR) images to the cub file format. These preprocessing steps are a necessary part of every pipeline discussed herein, since the reduced data record (RDR) images, which are widely available and immediately readable into GIS environments, lack the necessary geometric control for stereo processing. For CTX imagery, converting the EDR into a format suitable for stereo is a relatively simple process achieved via a sequence of ISIS commands. Image pairs are first converted to cube files using the ISIS mroctx2isis programme. Using spiceinit, the spice kernel for the cube files are then assigned, which contains the $X Y Z$ coordinates of the camera and its orientation in 3-D space. To improve the chance of stereo succeeding further down the pipeline, the images are individually radiometrically corrected using ctxcal. Finally, the images can be optionally destriped using ctxeven$o d d$, which removes any even or odd detector striping in the image. The images can then be processed further in ASP (Beyer et al., 2018).

Once the HiRISE and CTX images are in the appropriate cube format, pairs are ready to be ingested directly into stereo, the primary programme in the ASP suite. In practice, however, it is optimal to map project the imagery first. Map projecting the image pairs makes them as similar to one another as possible, which greatly increases the chances of subsequent pixel matching by the stereo algorithm (Beyer et al., 2018; Tao et al., 2018). The ASP cam2map4stereo.py script is based upon the ISIS cam2map function which takes spice kernel information and projects images according to a userdefined map projection. The ASP cam2map4stereo.py script does this with two input images with spice kernel information and, without further information, determines the overlap and the lowest-common resolution of each image. The two images are then map projected at identical resolution to this shared footprint. Map projection divorces the images from their associated camera models, and their position in 3-D space is now referenced with respect to map-projected co- 
ordinates (Beyer et al., 2018). This step is important, as the camera information for orbital imagery on Mars can be subject to large positional uncertainty. Map-projected images are then passed to stereo.

At its simplest, stereo takes the left and right overlapping images and by matching regions of pixels between them, outputs a point cloud that can then be processed further. Stereo is broken down into five steps.

Stage 0 is a preprocessing step that normalizes the mapprojected pair with respect to each other and, if nonprojected imagery were used as input, this is the stage at which images would be aligned based on one of several available algorithms. The primary purpose of this step is to remove any points present only in one image. Stage 0 outputs pre-aligned images and their respective image masks.

Stage 1 involves initial stereo correlation. The output from this stage is a disparity map: a three-channel image whose pixels describe the location of a pixel in the left image and the horizontal and vertical offset between that pixel and the matching pixel in the right image. Stereo attempts to find a matching pixel for all values not marked invalid by the Stage 0 masks. Correlation between large high-resolution image pairs is, therefore, computationally expensive, and stereo distributes the process between this and subsequent stages (Beyer et al., 2018). Stage 1 computes an initial guess correlation using a pyramidized search that is optimized for speed but suffers on resolution. To accelerate this process, first a box filter accumulator is applied, which minimizes repeat correlation operations. The pyramidized approach then refines the disparity estimates recursively at increasingly fine resolutions. Each pyramidized disparity search itself operates on sub-region tiles of comparable disparity from the preceding resolution, iteratively improving the correlation at each step (Beyer et al., 2018). The correlation window takes the form of a small rectangle that moves from the left image over the given search range in the right image. Matches between images are determined using a cost function operating in terms of disparity values, with the lowest cost match deemed best. Following Stage 1, each pixel in the disparity map will either have estimated disparity values assigned to it, or it will have been marked as invalid

Stage 2 is where a sub-pixel refinement algorithm is used to adjust valid pixels as marked by Stage 1. For the quickest results, the default sub-pixel-mode can be used to fit a simple 2-D parabola to points in an 8-pixelconnected neighbourhood around each pixel in the correlation cost surface (Beyer et al., 2018). The parabola's minimum is calculated and taken as the new sub-pixel disparity value.
Stage 3 addresses any outliers and matching failures generated by Stage 2 . Never will every pixel be matched between a stereo pair of images. Pixels may be rejected for any combination of several reasons: nonoverlapping areas between the two images; differences in image quality, lighting, contrast, and surface specular properties; particularly smooth surfaces with a low signal-noise ratio and without a sufficient level of texture for pixel correlation; and areas of distortion due to perspective differences between images, such as scarp walls and crater rims. Next, points with a triangulation error exceeding an automatically determined threshold are removed, and "holes" of failed pixels are left as is.

Stage 4 converts the disparity map into a 3-D point cloud. Stereo takes the ISIS cube information relating to the intrinsic and extrinsic camera information from each image. Combined, these parameters enable the "forward projection" of a 3-D point onto the imaging plane of the sensor in question. Once captured, however, it is not possible to forward project the route from an image pixel to the 3-D point (although it is possible to "back project" from the sensor to the original 3-D point). These are not, however, symmetrical operations and while one camera is sufficient to "image" a 3-D point on to a planar pixel, the opposite does not hold. To back project the route of a path from the image pixel to a 3-D point, it is necessary to have two paths from sensors both containing pixel locations which then converge at a point in 3-D space. Carried out across the entire scene, these ray intersections go on to form the basis of the output point cloud, describing the 3-D location of every matched pixel (Beyer et al., 2018).

Stage 5 is the final stage after stages $0-4$. Once the output point cloud has been generated by stereo, it is then passed to the ASP point2dem programme. This takes the point cloud and produces a GeoTIFF 32-bit floating point DEM in the GeoTIFF format which can then be exported. To deal with areas of limited points in the point cloud, point 2 dem includes several user-defined options for the treatment of holes in the output (Beyer et al., 2018). Stereo attempts to make every pixel between a left-right pair, and the raw point clouds describe the location of each of these pixels in 3-D space (Beyer et al., 2018). However, due to the nature of the Stereo process, and indeed more general-image matching, it is nearly impossible to match one individual pixel between two images. Instead pixel matching matches groups of pixels within a moving window. Each pixel therefore contains information, but the true resolution of the output DEM depends on several factors other than input image resolution. Accordingly, point clouds are generally downsampled by 3-4 times the input image resolution to improve the reliability of pixel matching results (Beyer et al., 2018). Hence, 1-2 $\mathrm{m} \mathrm{pixel}^{-1} \mathrm{DEMs}$ 
can be created from $25-50 \mathrm{~cm}_{\text {pixel }}{ }^{-1}$ HiRISE images and $\sim 20 \mathrm{~m} \mathrm{pixel}^{-1}$ DEMs from $\sim 5-6 \mathrm{~m} \mathrm{pixel}^{-1} \mathrm{CTX}$ imagery (Tao et al., 2018).

\subsection{Bundle adjustment}

It is possible to rapidly produce a rudimentary DEM - perfectly suited for terrain visualization or outreach purposes - by following the steps described in Sect. 2.1. Further, all workflows for producing DEMs from HiRISE and CTX imagery largely follow this same overall principle described above. However, to produce cartographic products fit for scientific use, additional considerations are required, and it is necessary to manage the errors arising from the Stereo process. A key source of these errors comes from uncertainty in the camera pointing information (Tao et al., 2018). Slight errors in the spice kernels assigned to each image mean that, in practice, rays from the two cameras do not intersect perfectly resulting in a triangulation error (Beyer et al., 2018). Generally, this error is managed by bundle adjustment. This is the process by which the properties of the cameras and the 3-D locations of the objects they image are adjusted simultaneously to minimize the error between back-projected pixel location and its actual measured location (Triggs et al., 2000). ASP, like most photogrammetric software, includes a means of bundle adjustment (Beyer et al., 2018). If disparity exists, the ASP bundle_adjust tool uses a least squares cost-function to rectify the positioning of cameras and thousands of points across the scene (Beyer et al., 2018). Bundle adjustment helps ensure that DEMs are internally consistent and well registered to other datasets (Beyer et al., 2018). Note that this triangulation error is not a true error, it refers only to the internal consistency of the DEM in question; high triangulation errors are always bad, whereas a low triangulation error means the DEM is internally consistent but may still be offset from the "true elevation" of the object in question. Adjusting for this error involves incorporation of external datasets, the geodetic locations of which have been determined a priori (Beyer et al., 2018).

\subsection{The Chicago method}

The "Chicago pipeline" (Mayer and Kite, 2016) uses only ISIS and ASP routines to produce HiRISE and CTX DEMs. CTX DEMs are crucial in the Chicago pipeline as they go on to serve as an intermediate data bridge to help co-register the HiRISE DEMs to lower-resolution datasets. CTX EDRs are prepared for ASP with ISIS routines that assign spice kernels and then correct the input images radiometrically. The ASP bundle_adjust tool is then used to minimize triangulation error and minimize artefacts in the final DEM. These bundleadjusted but non-map-projected images are passed to stereo, which generates a low-resolution initial DEM. The original CTX EDRs (prepared for the previous step) can then be passed to the ASP mapproject tool, alongside this initial low- resolution DEM onto which the images are projected. This helps ensure Stereo has the maximum probability of success on steep slopes. The map-projected images are then passed to stereo and point $2 \mathrm{dem}$, producing both a full-resolution DEM and a point cloud. The final step takes the output point cloud and uses the ASP $p c \_$align function to align the CTX point cloud to the MOLA point EDR (PEDR) data. A maximum displacement parameter is passed to the tool from inspection of the MOLA PEDR and the DEM from the previous step. Pc_align can use one of several least-cost algorithms to match two point clouds iteratively until they converge.

To produce the HiRISE DEMs, the Chicago pipeline follows the ASP pipeline described in Sect. 2.1, passing mapprojected images to stereo. The output point cloud, however, is then passed to $p c \_a l i g n$, along with the CTX-DEM created previously, taking advantage of the lower-resolution DEM to improve alignment of the HiRISE point cloud to the MOLA PEDR. As a laser-altimetry dataset, the MOLA PEDR is not subject to positioning errors of the same magnitude as other DEMs, and so it is taken as the "gold-standard" dataset to which other DEMs are aligned. The Chicago pipeline applies the complementary approaches of bundle adjustment and orthorectification to produce well-registered CTX DEMs, which in turn enable the production of wellregistered HiRISE DEMs. However, herein lies the difficulty with the widespread implementation of this pipeline: CTX stereo pairs are not always available for the areas covered by HiRISE pairs, and HiRISE DEMs generally have too small a footprint to intersect meaningfully with the often sparsely distributed MOLA PEDR data in the absence of a CTXresolution bridge.

\subsection{Stereo DEM production by the CASP-GO method}

The UCL "CASP-GO" pipeline (Tao et al., 2018) dispenses with the need for CTX DEMs entirely. This fully automated cloud-based pipeline for the global production of CTX and HiRISE DEMs instead uses CTX orthorectified images to co-register HiRISE pairs. To co-register these CTX pairs, points extracted from orthorectified HRSC images are used. These, together with a tie-point-based multi-resolution image co-registration scheme, guarantee global geo-referencing compliance with HRSC, and by extension MOLA, DEMs. The UCL pipeline makes use of both ISIS-ASP functions - including the implementation of an initial rough disparity map and the use of the Bayes expectation maximization (BEM) sub-pixel affine adaptive matcher - but then supplements and refines the outputs using Gotcha, a 5th generation least squares matcher (Shin and Muller, 2012), and other proprietary algorithms. One of the stated goals of the UCL pipeline is to incorporate the CASP-GO pipeline into the online $i$-Mars GIS project (https://bit.ly/2UrVKdT, last access: 26 February 2019) and release processed DEMs through the PDS ODE repository. However, to date only a portion of the planned CTX DEMs have been made available on $i$-Mars, 
and no HiRISE DEMs have been processed. Furthermore, the underlying algorithms and their exact implementation have not yet been made open-source.

\section{Development of near-automatic open-source AU workflow}

Each of the open-source pipelines for the production of HiRISE and CTX DEMs summarized above either lacks suitable geometric control, is built upon algorithms yet to be made publicly available, or depends on data that have limited global coverage. Here, we present a new near-automatic and relatively rapid $(\sim 24 \mathrm{~h})$ open-source AU workflow for the production of HiRISE DEMs. Making use of the globally widespread HRSC (Jaumann et al., 2007) and MOLA DEMs (Smith et al., 2001), and existing - and open-source - sub-routines, this workflow can be used to produced geometrically well-registered DEMs of a quality comparable to those released on the PDS ODE to date.

The AU pipeline we present (Fig. 2) is based entirely upon the ASP 2.6.1 (available from: https://go.nasa.gov/2NIk74m, last access: 26 November 2019) and ISIS 3.5.3 (available from: https://bit.ly/2C3jBcE, last access: 10 June 2016) programmes. HiRISE EDRs are prepared for ingestion into ASP using the routines described above (Sect. 2.1), passed to bundle_adjust, and then map projected in reference to a HRSC DEM. The map-projected stereo image pair are then passed to stereo, and matching is carried out using the BEM subpixel refinement routine. Finally, this point cloud is then aligned to the HRSC DEM, before being converted into the DEM format for further study. Below, we detail this pipeline and evaluate output DEMs by comparing them to several DEM products already available (Sect. 3.2).

The AU pipeline presented here is designed to enable a wide range of researchers to create and use 1-2 m resolution HiRISE DEMs using the well-documented ASP and ISIS programmes. The AU pipeline removes the need to wait for stereo pairs to be processed and released on the PDS ODE repository. With only minor modifications, the following $\mathrm{AU}$ pipeline can also be used to create CTX DEMs, although these are not discussed here.

\subsection{The AU method}

The first stage after preprocessing (Sect. 2.1) is to bundle adjust the unprojected left and right image pairs. ASP's bundle_adjust tool allows the input of user-selected ground control points (GCPs: Fig. 3) matched between the (unprojected) left and right images, a lower-resolution, but map projected, reference image used to assign $X Y$ coordinates to each point, and an existing DEM from which the elevation $(Z)$ information of each GCP can be taken. For HiRISE images, a CTX EDR image and a HRSC DEM are used (for producing CTX DEMs, one would use HRSC imagery and the HRSC DEM).
To ensure co-registration between the CTX and HRSC images used in the bundle adjustment of HiRISE pairs, the CTX EDR is converted to an ASP-appropriate format and then orthorectified and map projected onto the HRSC DEM with the ASP map_project tool.

GCPs are then picked on HiRISE stereo pairs. At least 30 well-distributed GCPs corresponding to identical features (such as rock outcrops and crater rims) are matched between all three images (Fig. 3) using the graphical user interface version of stereo (stereo_gui). Once GCPs are identified, the HRSC DEM is then selected, and a text file containing the 3-D coordinates of each GCP is written. This GCP list is then passed to bundle_adjust alongside the unprojected stereo pair.

Bundle_adjust is built upon the Ceres Solver - an opensource least squares minimization algorithm from Google which iterates through a lowest-cost function to minimize the triangulation error between the positions of GCPs as they appear in the unprojected images and the position of the GCPs as measured from the external geo-referenced image and DEM. Uncorrected, these uncertainties would result in systematic errors in the global position of the output DEM and local slope, as well as local distortions and warping. By bundle adjusting the 3-D positions of the stereo camera as well as that of thousands of similar constraints, inconsistency between external geodetic control networks is kept to a minimum, and the internal consistency of the output DEM itself is improved. Bundle_adjust generates a series of transformation values, which are then passed on to map_project and stereo in subsequent steps.

Following bundle adjustment, images are projected using ASPs map_project tool. Making each image as similar as possible to each other ensures the highest chance of success for subsequent processing. Map_project takes the left and right camera images together with the bundle adjustment solution and orthorectifies images onto a low-resolution DEM. Here, we use the $d t 4$ HRSC DEM product, a map projected $75 \mathrm{~m} \mathrm{pixel}^{-1}$ DEM referenced to the Mars ellipsoid. Mapprojection onto a DEM, rather than a datum, helps mitigate the impact of particularly steep terrain which is extremely difficult to match between stereo images. Map projection leaves only very small differences in perspective between images, maximizing the efficacy of stereo.

Pixel matching between the two map-projected images then follows the same stereo procedure as described in Sect. 2.1 but with several additions to the command line input. The most notable of these is the selected sub-pixel refinement strategy. Rather than the default parabolic subpixel refinement algorithm, here we use the BEM weighted affine adaptive window correlation. Featuring a deformable search window that can be rotated, scaled and translated as it searches for pixels; this adaptive algorithm is especially suited for accurate matches on steep terrain, where significant foreshortening can cause perspective distortion, which is detrimental to matching. Based on the Lucas-Kanade tem- 


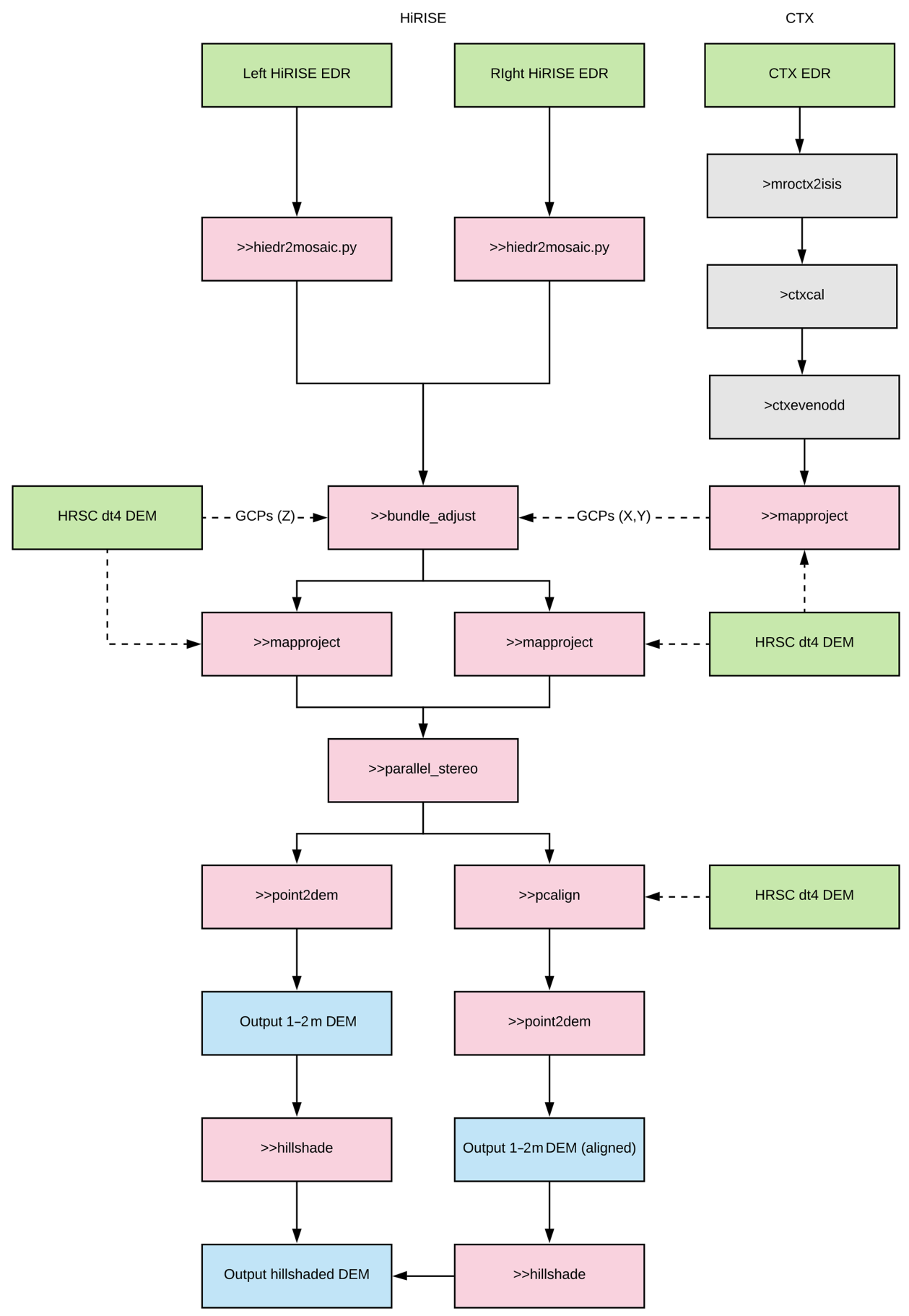

Figure 2. The workflow presented here for the production of 1-2 $\mathrm{m} \mathrm{pixel}^{-1}$ HiRISE DEMs from stereo image pairs. Green steps represent PDS ODE data input, grey indicate ISIS commands, pink represent ASP steps, and blue represent output data.

plate tracking algorithm (Baker and Matthews, 2004; Nefian et al., 2009; Beyer et al., 2018), the ASP Bayes EM treats the Lucas-Kanade variables as random parameters within an expectation maximization (EM) framework. A Gaussian mixture component is also included, which provides a degree of robustness against image noise (Beyer et al., 2018). This Gaussian mixture component also weighs the sub-pixel correlation kernel thereby reducing its effective size. Here, instead of the default 35 by 35 sub-pixel kernel window, we use a sub-pixel kernel size of 55-75 by 55-75 pixels to account for this weighting.

Following stereo, the point cloud is converted to a DEM and is compared to the HRSC DEM. The maximum offset between the two is then fed to $p c \_a l i g n$, as an initial estima- 


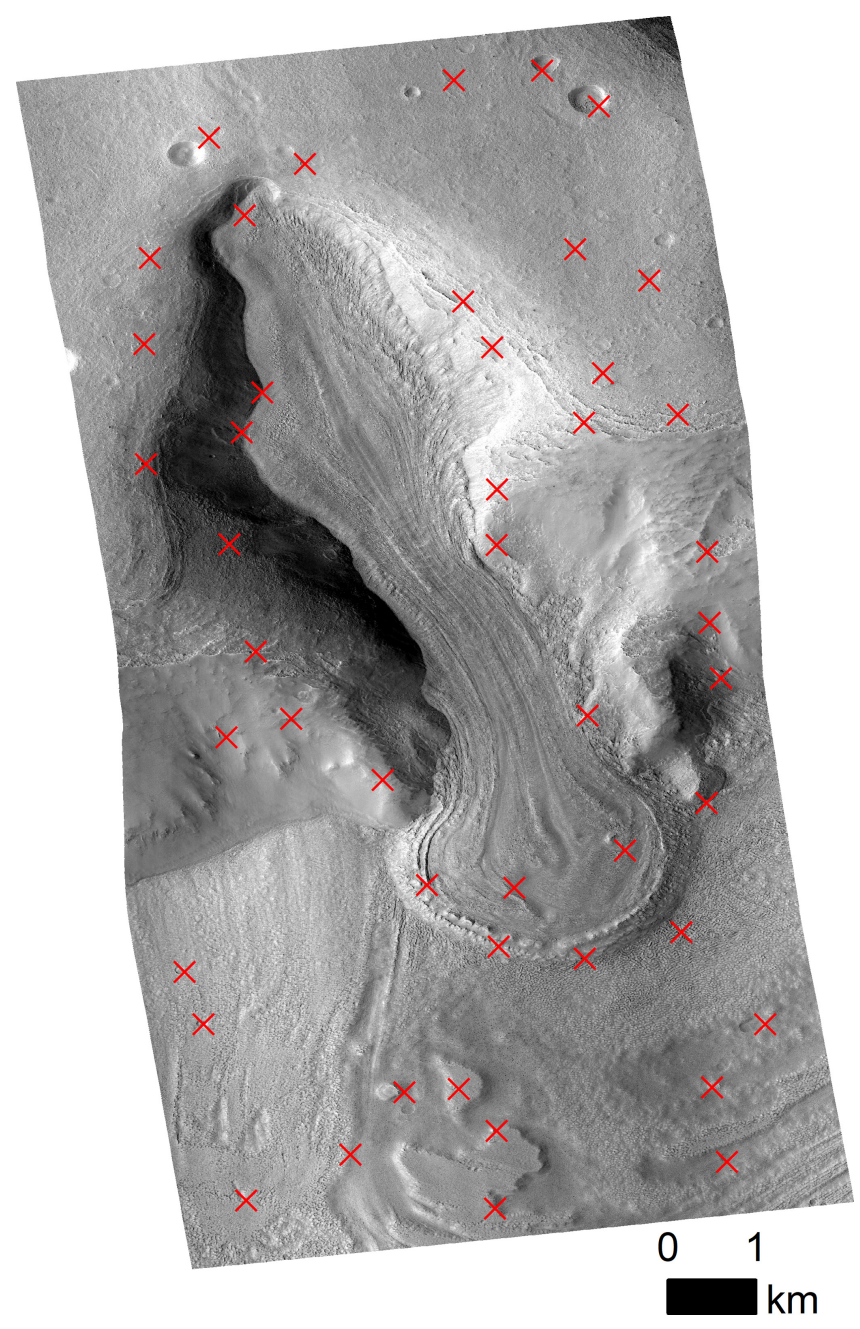

Figure 3. An example ground-control point network (red crosses: $n=35)$ selected between the left (ESP_052535_2225) and right (ESP_052324_2225) images which form AU DEM 4 (Fig. 1), a map-projected CTX image (P22_009653_1487_XI_31S300W), and the HRSC DEM (h1523_0000_dt4) using stereo_gui in ASP. Note, points are widely distributed throughout the image.

tion of the magnitude of transformation necessary to align the output point cloud and the HRSC DEM. Here, with DEMs significantly offset $(>100 \mathrm{~m}$ ), we found it optimal to use two different alignment algorithms in sequence. First, the initial transform is carried out with the point-to-plane iterative closest point algorithm, which solves for a combination of rotation and translation. Once the point clouds are as closely aligned as possible, the transformation obtained from the previous alignment is fed to $p c \_a l i g n$ and the similarity least squares transformation is then used, solving for rotation and translation alignment as well as scale. For the similarity least squares to converge, the transformation needs to be already extremely close. For point clouds that are already close prior to alignment, the point-to-plane ICP transformation alone may suffice. As we go on to describe in Sect. 4, the use of $p c \_a l i g n$ does not always improve the alignment of DEMs and can degrade the alignment between the output DEM and the HRSC DEM. However, because $p c \_a l i g n$ outputs a new DEM and leaves the input DEM unchanged, best practice remains to make use of the tool and assess DEM outputs visually.

\subsection{Evaluating the AU pipeline}

High-resolution DEMs on Earth are generally assessed by comparison with the coordinates of fixed points within the DEM's footprint. Quantitative comparison of the deviation between the surface as represented by the DEM and the surface represented by the in situ points serves as a measure of DEM accuracy. However, the "ground truth" is largely unknown on Mars. In the pipeline presented here, GCPs are marked between stereo pairs, a third geo-referenced image, and a low-resolution DEM. However, the 3-D coordinates of GCPs are themselves subject to numerous uncertainties, and hence cannot be used to assess the stereo DEMs representation of ground truth, a problem that applies to all Mars DEMs. In order to evaluate the uncertainty in the DEMs produced by our pipeline, we calculated the root mean square error (RMSE) between HiRISE AU DEMs and those already available from the PDS ODE, both against each other and against the lower-resolution HRSC and MOLA DEMs.

Here, we create and assess four DEMs using the AU pipeline. Each stereo pair was selected based on the availability of pre-existing PDS HiRISE DEM (PDS DEM hereafter) made from a different stereo pair whose footprints partially or totally intersected those of the stereo pair we processed. The four AU DEMs created were made in reference to the widely used $D \_$mars datum value of $3396.19 \mathrm{~km}$. The HRSC DEMs, however, are referenced to a value of $3396 \mathrm{~km}$, and so for comparison our DEMs were adjusted by $190 \mathrm{~m}$. This adjustment was only made post-production to allow comparison to the HRSC product, but this adjustment can be made at several steps in the AU pipeline if desired. Furthermore, DEMs can be created in reference to either the datum ellipsoid or the equipotential areoid (geoid on Earth). The HRSC DEMs, for example, come in both $d a 4$ (areoid) and $d t 4$ (ellipsoid) versions. The ASP includes a tool (dem_geoid) for converting these datums. Conversion allows comparison between our DEMs, PDS HiRISE DEMs (referenced to a local datum and therefore effectively, the areoid), and the $d a 4$ HRSC DEMs. In total, for the purposes of quality assessment, we produced four AU DEMs from each stereo pair; two DEMs referenced to the ellipsoid and areoid from each of the point clouds before and after the $p c$ _align step in the workflow described above (Sect. 3.1).

\subsubsection{Comparison 1: HiRISE-HiRISE RMSE}

RMSE was first calculated between HiRISE DEMs created here, and those already available on the PDS ODE repository, 
using the following equation:

$\operatorname{RMSE}=\sqrt{\left(Z_{X 1}-Z_{X 2}\right)^{2}}$,

where $X 1$ is the reference PDS DEM, and $X 2$ is the comparison AU DEM produced here. DEMs were reprojected to a common equirectangular projection using a bilinear interpolation procedure. To reduce the computational strain of working with $1 \mathrm{~m} \mathrm{pixel}^{-1} \mathrm{DEMs}$ (each containing $>1.5 \times 10^{8}$ individual cells), DEMs were downsampled to a $10 \mathrm{mpixel}^{-1}$ resolution. Downsampling by this factor also helps to reduce noise and/or artefacts present in DEMs at their native $1 \mathrm{~m} \mathrm{pixel}^{-1}$ resolution.

Even when downsampled to $10 \mathrm{~m}$ resolution, lateral differences in the projected footprint of each HiRISE DEM has the potential to greatly affect the calculated vertical error, especially in areas of steep terrain. Further, the PDS HiRISE DEMs are subject to the same positioning uncertainties (though not necessarily of the same magnitude) as DEMs produced elsewhere, and so it may be difficult to determine which DEM is the more accurate representation. This issue is further compounded by the large resolution gap between the HiRISE DEMs and other DEMs. This makes matching features extremely difficult, especially in variable topography where no clear feature may be easily distinguished. To ensure that the primary component of RMSE is vertical differences between DEMs, we excluded pixels with slopes $>10^{\circ}$. The lope was calculated using the ArcGIS (v.10.5) slope tool, with each DEM reprojected into a length-preserving mercator projection. Slope layers were then projected back into the equirectangular projection, and cell values were retrieved. RMSE was calculated between PDS DEMs and the AU DEMs produced here both before and after slope thresholding.

\subsubsection{Comparison 2: HiRISE-HRSC RMSE}

The RMSEs of both PDS DEMs and AU DEMs were calculated against the HRSC ( $d a 4$ and $d t 4$ ) DEMs, representing the next highest resolution publicly available DEM product (75-250 m). Again, all DEMs were reprojected to a common equirectangular projection. Each HiRISE DEM was then downsampled to match the resolution of the HRSC DEM. The RMSE of each HiRISE DEM against the HRSC DEM could then be calculated using Equ. (1) with the low-resolution reference HRSC DEM and the comparison HiRISE DEM substituted for the $X 1$ and $X 2$ terms respectively.

\subsubsection{Comparison 3: HiRISE-MOLA RMSE}

AU DEMs were also compared to MOLA individual shot data (PEDR). PDS DEMs are made in reference to this MOLA dataset, with a reported average RMSE of $1 \mathrm{~m}$ between the two DEMs. For comparison, the RMSE of each of our AU DEMs and the PDS DEM was calculated against the
MOLA PEDR data. Each MOLA shot has a vertical uncertainty of $1.5 \mathrm{~m}$ but a horizontal uncertainty of approximately $100 \mathrm{~m}$. To account for this, a buffer of $50 \mathrm{~m}$ radius was applied to each point. Each buffered point was then rasterized to the same $10 \mathrm{~m}$ resolution as each of the HiRISE DEMs. The RMSE between the MOLA PEDR and each HiRISE DEM could then be calculated using Eq. (1) and substituting the MOLA PEDR for the $X 1$ term.

\subsubsection{Comparison 4: MOLA-aligned HiRISE-MOLA RMSE}

Because PDS DEMs are made in reference to the MOLA PEDR data, the RMSE was also calculated against a AU DEM created from a point cloud aligned to the MOLA PEDR data using $p c \_a l i g n$. This was compared to the RMSE of the PDS DEM against the MOLA PEDR and the RMSE of AU DEMs made without any MOLA input.

\subsection{DEM artefacts}

Finally, visual observation of the output DEMs is a useful means of evaluating their quality. Processing artefacts - evidently erroneous features present in a DEM that are absent in the actual terrain - are visible in both PDS and AU DEMs (Fig. 4). These artefacts are best visualized in hillshaded images produced from DEMs (Fig. 4a-c) but are also clear in surface slope maps (Fig. 4d). Artefacts are predominantly due to the nature of the HiRISE images, which comprise 10 individual bands from separate charge-coupled devices (CCDs) (Fig. 4a-b). Longitudinal lineations visible in some PDS and AU DEMs with hillshading (e.g DTEEC_028591_1880_028657_1880_101: https:// bit.ly/2DOFAE2, last access: 10 February 2019) are a direct result of the stitching together of these bands. Other artefacts are present in both PDS and ASP DEMs, including various failures due to lighting conditions and low-texture terrains, several examples of which are illustrated in Fig. 4. Cursory visual analysis of several DEMs indicates that ASP DEMs produced here appear less affected by artefacts than HiRISE PDS DEMs. However, in areas of especially low-terrain texture, the ASP-matching algorithm was less successful than the SOCET-SET pixel matcher used to create PDS DEMs (Figs. 4a and A2 in the Appendix). Increasing the sub-pixel kernel size can increase the efficacy of stereo (Beyer et al., 2018) and, although not implemented here, implementation of the semi-global-matching algorithm in stereo can also improve pixel matching in low-texture terrains (Beyer et al., 2018).

\section{Case study - evaluating AU DEM 2}

Our workflow was successfully used to generate numerous DEMs across the surface of Mars, including four which we evaluate against other datasets. For each of these four sites 


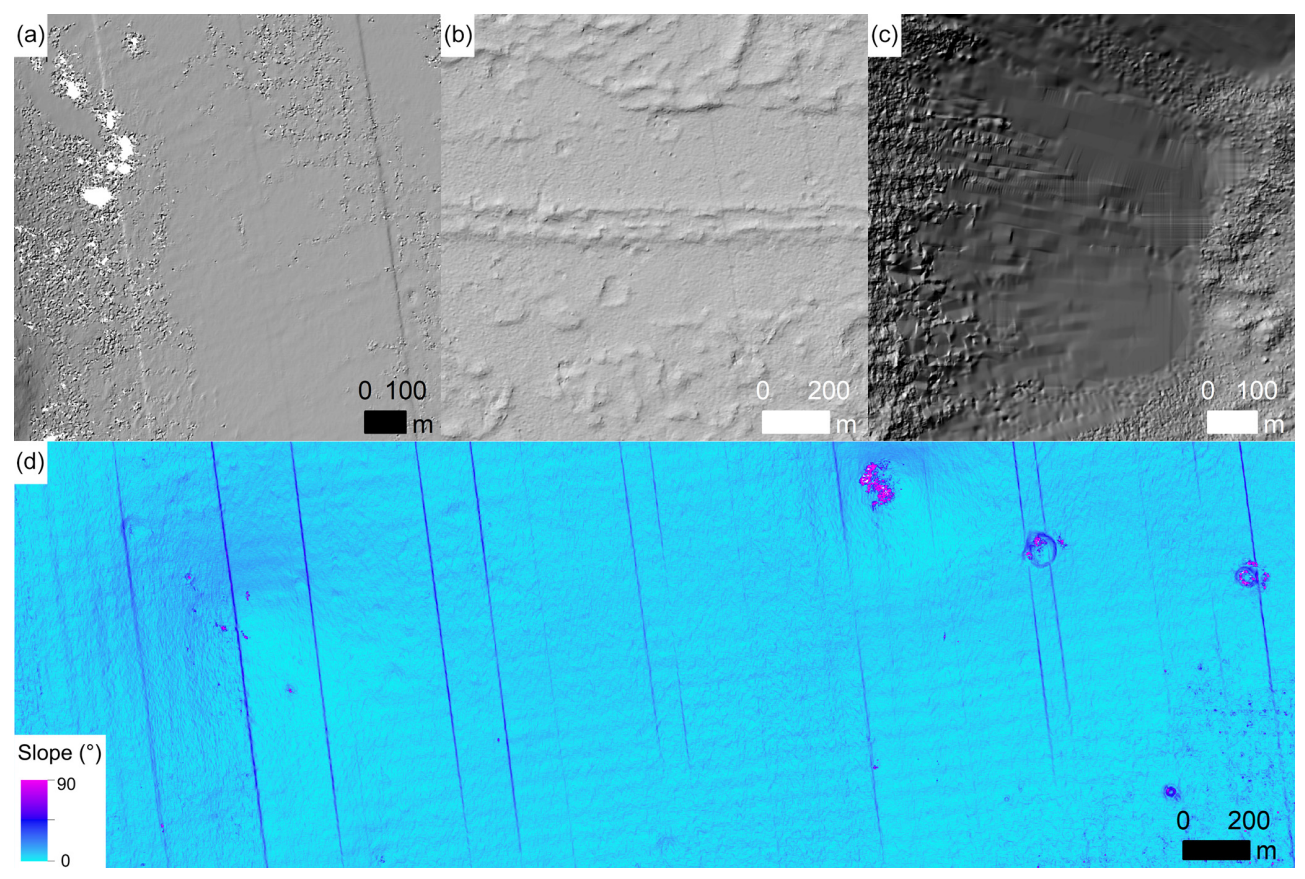

Figure 4. Several visible artefacts present in AU and PDS HiRISE DEMs. (a) Pixel-matching failures associated with low-terrain texture in AU DEM 3. Longitudinal foliations are also visible and are more clearly seen in (d). These foliations are not clearly visible in either input image, but arise in the DEM because HiRISE images consist of 14 individual bands, 10 of which must be stitched together to create a single image. (b) Further longitudinal foliations associated with individual HiRISE bands, this time present in the PDS DEM for site 1 (DTEEC_002118_1510_003608_1510_A01: https://bit.ly/2tSD8Ig, last access: 1 February 2019). (c) Artificial smoothing present in the site 1 PDS DEM. In lieu of pixel-matching information, the SOCET-SET algorithm has been used to fill in the data gaps, resulting in the smooth artefact. In the ASP workflow presented here, gaps in data due to pixel failure are left as is, leaving the empty pixels visible in (a). (d) Longitudinal foliations clearly visible in a slope map generated from AU DEM 3.

(sites 1-4 hereafter), one AU DEM was generated. RMSE was calculated between AU DEMs 1-4, a PDS DEM, HRSC DEM, and a MOLA PEDR DEM. The RMSE between AU DEMs 1-4 and pre-existing PDS, HRSC and MOLA DEMs are reported in Table 1. At each site, AU DEMs 1-4 compare closely to other DEMs. The lowest RMSE reported here from an AU DEM produced using our workflow is $9.75 \mathrm{~m}$ (Table 1). Further, the AU DEMs produced here represent a major increase in resolution over HRSC (Fig. 6a-b) and MOLA DEMs.

Of the four sites for which DEMs were created here, site 2 includes the most geologically complex landform assemblage (Fig. 5), and AU DEM 2 serves as a useful illustration of common issues and details pertaining to all four AU DEMs. The discussion herein, therefore, is primarily focussed on AU DEM 2, and for the details specific to AU DEMs 1, 3, and 4 the reader is referred to the Appendix.

AU DEM 2 was created here using the HiRISE images: ESP_032115_1685 (left image) and ESP_031838_1685 (right image). This stereo pair intersects with the eastern portion of DTEEC_003910_1685_005400_1685_U01, a PDS DEM on the PDS ODE repository (https://bit.ly/2tQr89U, last access: 9 February 2019). Both DEMs show a region of layered terrain in a Noctis Labyrinthus pit (Fig. 5) with over $300 \mathrm{~m}$ of exposed light- and dark-toned materials, including exposed hydrated mineral units. This region of Mars, towards the western limit of Valles Marineris, is of interest to those studying the planet's aqueous history (e.g Weitz et al., 2011). The landform assemblage within site 2 consists of a flat bottomed pit containing low-amplitude aeolian landforms, flanked by a steep-sided cliff-portion in the north and a more gradual slope in the south (Figs. 5 and 6a-b). Site 2 includes a complex mixture of terrains, surface textures, and gradients (Fig. 5). It is, therefore, an ideal environment in which to test our pipeline for the production of AU DEMs from HiRISE images.

Comparison one yielded a lowest RMSE of $25.8 \mathrm{~m}$ between post-pc_align AU DEM 2 and PDS DEM once slopes $>10^{\circ}$ were removed (Table 1 ). With these steep slopes included, the RMSE increased to $39.77 \mathrm{~m}$ (Table 1). Before the application of $p c \_$align, the RMSE of AU DEM 2 was $62.38 \mathrm{~m}$ before slope removal and $38.54 \mathrm{~m}$ after removal (Table 1). AU DEM 2 before $p c \_a l i g n$ was horizontally closely registered to the PDS DEM (Fig. 6a), and alignment shifted the AU DEM eastwards with a strong rotational component to movement (Fig. 6a and e). The RMSEs of both AU DEM 2 and the PDS DEM were also calculated against HRSC DEM scene h1999_0000 (comparison 2). It was anticipated that the 


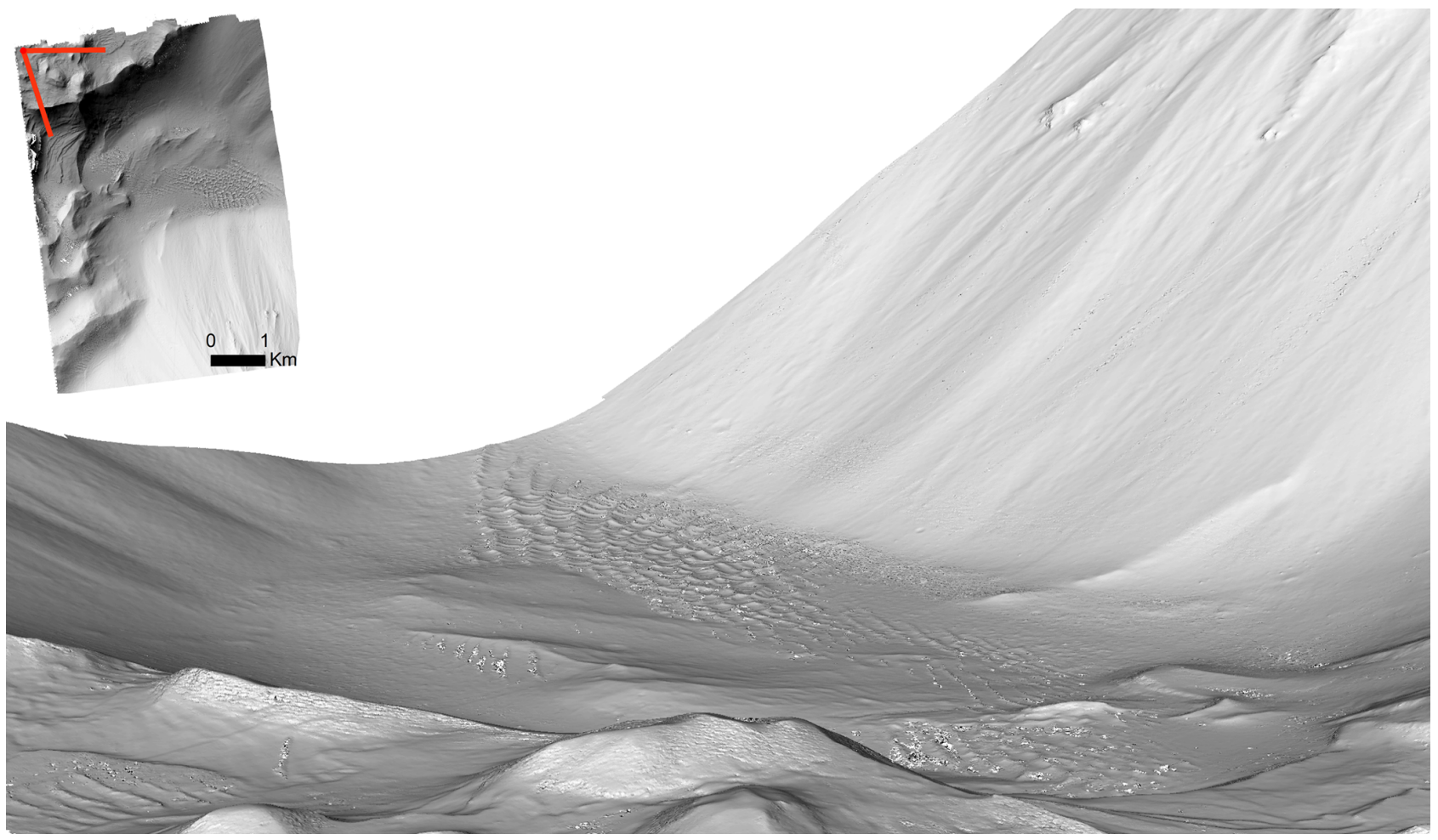

Figure 5. Oblique view of a region of layered terrain in a Noctis Labyrinthus pit (site 2). A complex landform assemblage is visible, including aeolian dunes, scarps, and hill-slope processes. View generated from a $2 \mathrm{~m} \mathrm{pixel}^{-1}$ hillshade of AU DEM 2, draped over the AU DEM itself and visualized in ArcScene. Inset map shows AU DEM 2 hillshade in plan view, approximate viewpoint marked in red.

RMSE of our AU DEMs against the HRSC DEMs would be of similar magnitude to the PDS DEMs (Table 1). The $p c \_a l i g n$ step appears to have improved the co-registration of AU DEM 2 with the HRSC DEM, and the RMSE before and after $p c \_a l i g n$ was 79.28 and $41.03 \mathrm{~m}$ respectively (Table 1). By comparison, the PDS DEM had an RMSE of $47.49 \mathrm{~m}$ against the HRSC DEM (Table 1). Finally, the RMSE of AU DEM 2 and the PDS DEM was compared against the MOLA PEDR data (Comparison 3 and 4: Table 1). As before, $p c$ align reduced the RMSE from 64.63 to $41.23 \mathrm{~m}$ against the reference (MOLA) dataset. The RMSE of the PDS DEM against the MOLA PEDR, in reference to which it was created, was $46.61 \mathrm{~m}$. RMSE fell to $35.32 \mathrm{~m}$ once the $p c \_a l i g n$ step was repeated on AU DEM 2 with the MOLA PEDR in place of the HRSC $d t 4$ product (Table 1).

After $p c$ align, DEM 2 had a lower RMSE against the HRSC and MOLA datasets with which it was compared than with the PDS DEM. Pc_align also lowered the RMSE between the two HiRISE products. For each of the four AU DEMs produced and tested here, an RMSE was calculated before and after point cloud alignment. As shown by Table 1, in each of the other three cases reported here, the RMSE of the ASP DEM pre-alignment was much lower than, or very similar to, the RMSE of the ASP DEM post-alignment when both are compared to the reference DEM, be it PDS HiRISE, HRSC, or MOLA data.
The RMSE of AU DEM 2 is notably higher when compared to the PDS DEM than the RMSE of the other three AU DEMs. AU DEM 2 also showed the largest decrease in RMSE when steep slopes were excluded. Together, these indicate that AU DEM 2 was poorly co-registered with the PDS HiRISE DEM with which it was compared (Fig. 6e). In contrast, visual analysis of AU DEM 2 after $p c \_a l i g n$ indicates it is better co-registered with the HRSC DEM than the PDS DEM (Fig. 6a-b), and a higher RMSE between the PDS and HRSC DEMs supports this conclusion (Table 1). This demonstrates the ability of our open-source workflow to create well-registered DEMs even for challenging terrain types; in this instance matching pixels well despite a complex landform assemblage consisting of steep, shadowed, and low-texture terrain.

AU DEM 2 was the only DEM produced here for which $p c \_a l i g n$ reduced the RMSE against reference datasets. Each of the other three AU DEMs all registered closely without the $p c \_a l i g n$ step (Figs. A2 and A3 in the Appendix). In fact, in each of these cases, point cloud alignment either shifted the output DEM dramatically or not at all (Fig. A4), with a corresponding change or lack thereof in the RMSE values calculated (Table 1). We conclude that operator discretion should be exercised when deciding whether or not to implement the final $p c \_a l i g n$ step in this workflow. In some instances, point cloud alignment can actually degrade the accuracy of 


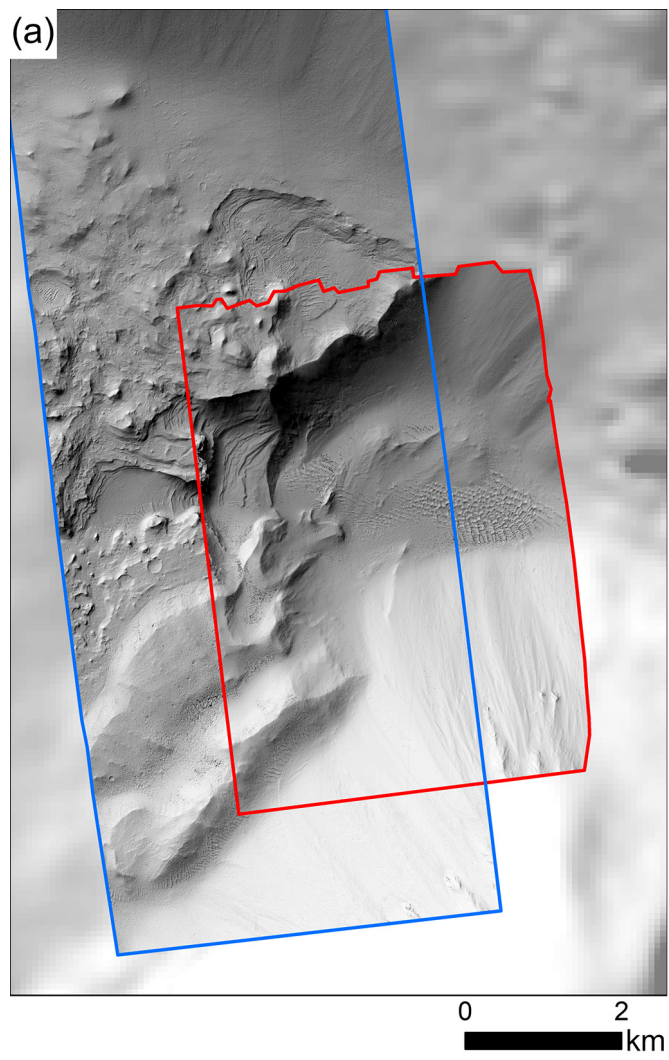

(c)

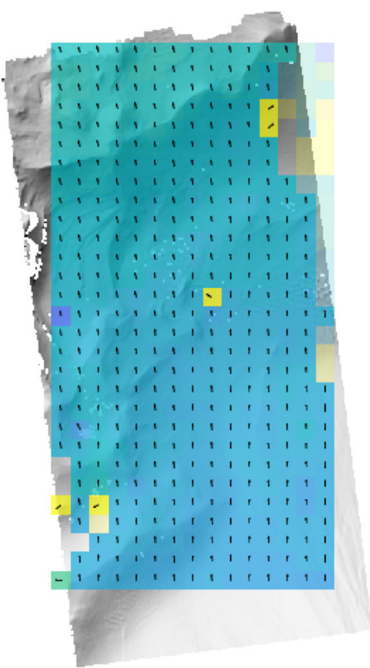

(d)

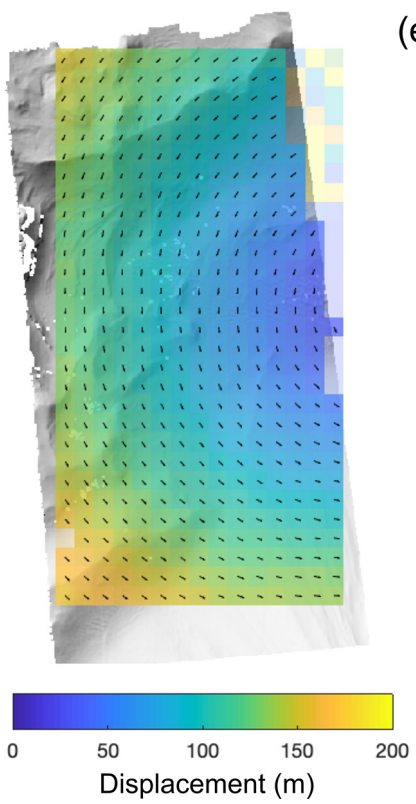

(b)

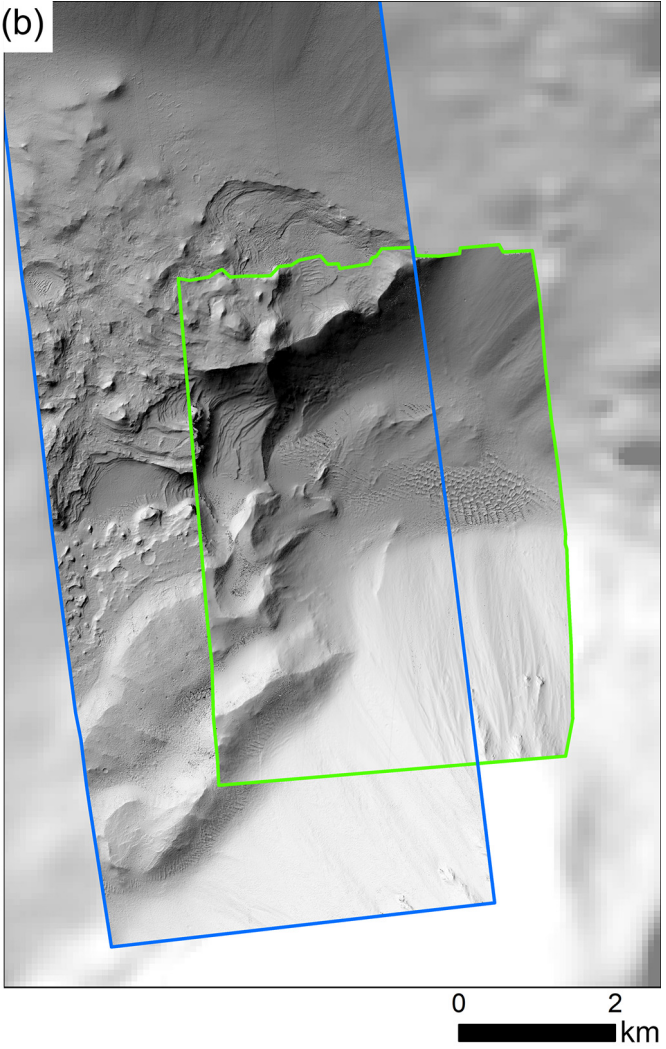

(e)

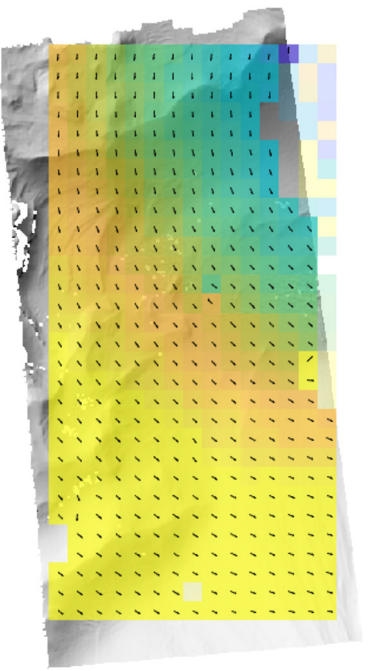

Figure 6. Alignment of site 2 DEMs including the AU DEM produced using the ASP pipeline presented, the PDS DEM (DTEEC_003910_1685_005400_1685_U01: https://bit.ly/2tQr89U, last access: 10 February 2019), and the HRSC DEM (h1999_0000_da4). (a-b) Hillshaded PDS DEM (blue box), the AU DEM 3 pre-pc_align (red box), and AU DEM 3 post-pc_align (green box). Background is the hillshaded HRSC DEM. Note the misalignment between AU and PDS DEMs. (c) Displacement between the AU (pre-pc_align) DEM and the PDS DEM. Little displacement indicates strong agreement. (d) Displacement measured between the two AU DEMs. (e) Displacement between the AU (post-pc_align) DEM and the PDS DEM. Note the strong rotational component to displacement as the AU DEM shifts to better match the HRSC DEM. Panels (c-e) created using the ImGRAFT feature tracking algorithm (Messerli and Grinsted, 2015). 


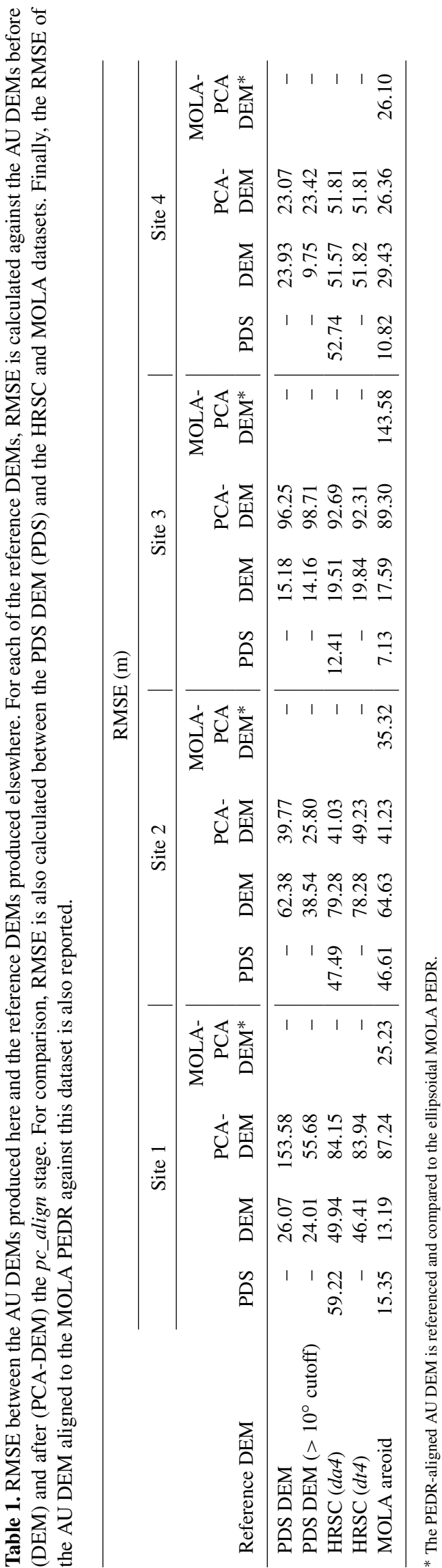

the AU DEMs produced using our pipeline. Comparison of DEMs from multiple sources both visually and qualitatively as done here, as well as comparison to geo-referenced images, is therefore recommended.

\section{Summary and conclusions}

Our AU workflow with the open-source ASP produces DEMs of similar quality to those already available, enabling the relatively quick $(\sim 24 \mathrm{~h}$ on a research computing cluster) and largely automatic production of $1-2 \mathrm{~m}$ resolution DEMs in areas that at present do not have comparable highresolution coverage. With a lowest RMSE of $\sim 9 \mathrm{~m}$ against HiRISE DEMs already publicly available, this workflow represents a valuable and easily implemented means of expanding the DEM coverage of Mars with outputs of similar quality to existing products. Further, our method is entirely based upon free open-source software and data, with no need for proprietary algorithms or intermediate CTX DEMs. This workflow has largely been implemented on the Supercomputing Wales research computing cluster, making use of the parallelized implementation of stereo (parallel_stereo) provided in the ASP bundle. However, this workflow has been successfully run (albeit more slowly: $\sim 72 \mathrm{~h}$ ) on a consumergrade Unix laptop computer with little need for parameter adjustment. By making use of open-source well-documented programmes and data, the AU pipeline is relatively simple to use and is therefore ideal for the quick generation of several DEMs within a study site or area of interest.

Code and data availability. All code and programmes used in this study are freely available online. The latest version of the Ames Stereo Pipeline is available at: https://ti.arc.nasa.gov/tech/ asr/groups/intelligent-robotics/ngt/stereo/ (last access: 26 November 2019), the Integrated Software for Imagers and Spectrometers is available at: https://isis.astrogeology.usgs.gov/ (last access: 10 June 2019), and all data used in the analysis are available from: http://ode.rsl.wustl.edu/ (last access: 26 November 2019). Output DEMs created for this study are available from the corresponding author on request, as are example input scripts. 


\section{Appendix A: Comparison of AU DEMs 1, 3 and 4}

\section{A1 AU DEM 1}

Site 1 is the western ejecta blanket of Zumba crater, a $\sim 3 \mathrm{~km}$ wide, young, and rayed impact crater in Daedalia Planum (Chuang et al., 2016). The HiRISE stereo pair ESP_020486_1510 (left) and ESP_014262_1510 (right) was used to create AU DEM 1. One PDS DEM (DTEEC_002118_1510_003608_1510_A01: https:// bit.ly/2tSD8Ig, last access: 10 February 2019) overlaps with AU DEM 1 (Fig. A2). The HRSC DEM for site 1 is h2538_0000.

The RMSE of comparison 1 was $26.07 \mathrm{~m}$ before slopes $>10^{\circ}$ were removed, and this lowered to $24.01 \mathrm{~m}$ once these slopes were excluded. At site $1 p c \_$align shifted the AU DEM dramatically (Figs. A1 and A4). As a result, RMSE between AU 1 and the PDS DEM increased to 153.58, and $55.68 \mathrm{~m}$ once slopes $>10^{\circ}$ were removed. This large increase in RMSE (and $\sim 100 \mathrm{~m}$ difference before and after slope removal) corroborates visual analysis and indicate $p c$ align degraded the alignment of AU DEM 1 relative to the PDS DEM.

The RMSE of AU DEM 1 against the HRSC da4 DEM was $49.94 \mathrm{~m}$ before and $84.15 \mathrm{~m}$ after $p c \_a l i g n$. The RMSE of PDS DEM was $59.22 \mathrm{~m}$. Together, these indicate that AU DEM 1 offered improved accuracy relative to the PDS DEM. However, it is worth noting the different footprints of each DEM, the PDS DEM includes the full diameter of Zumba crater, and AU DEM 1 only covers the western portion. A steep-walled crater represents a challenging terrain for stereo, and this may account for the higher RMSE of the PDS DEM.

Finally, the RMSE against the MOLA PEDR of AU DEM 1 and the PDS DEM compare closely at 13.19 and $15.35 \mathrm{~m}$ respectively. As with the other comparisons, $p c \_a l i g n$ increased the RMSE of the AU DEM against the MOLA PEDR. Pc_align did lower the RMSE of AU DEM when the MOLA PEDR was used as the alignment DEM, but at $25.23 \mathrm{~m}$ this was still $10 \mathrm{~m}$ higher than the AU DEM with no alignment.

\section{A2 AU DEM 3}

Site 3 is a largely flat low-texture terrain, and includes the western rim of Endeavour crater, a Noachian-aged crater within Meridiani Planum, Arabia Terra (Mittlefehldt et al., 2018; Stein et al., 2018). AU DEM 3 was created using PSP_010341_1775 (left) and PSP_010486_1775 (right), a HiRISE image pair taken to assist in planning for the Mars Exploration Rover Opportunity's long-range traverse. DTEEC_018701_1775_018846_1775_U01 (https: //bit.ly/2TrxFXX, last access: 10 February 2019) is the site 3 PDS DEM (Fig. A2), and h3198_0001 is the HRSC DEM.
At site 3, the RMSE of comparison 1 was 15.18 , and $14.16 \mathrm{~m}$ once slopes $>10^{\circ}$ were removed. Pc_align increased this RMSE to 96.25 and $98.71 \mathrm{~m}$ once slopes $>10^{\circ}$ were removed. The RMSE of AU DEM 3 prior to $p c \_$align is the second lowest RMSE recorded against the PDS DEM reference (Table 1 in the main text), suggesting the two DEMs were extremely well co-registered (Fig. A2). At site 3, pc_align again resulted in a large shift (Fig. A4) and degraded the accuracy of the AU DEM in reference to the PDS DEM.

AU DEM 3 also appeared well co-registered with the HRSC DEM without the pc_align step. Comparison 2 against the HRSC da4 DEM yielded an RMSE of $12.41 \mathrm{~m}$ for the PDS DEM, $19.51 \mathrm{~m}$ for AU DEM 3 pre-pc_align and $92.69 \mathrm{~m}$ for AU DEM 3 post-pc_align. Comparisons 3 and 4 returned similar results, and the RMSE in reference to the MOLA PEDR of the PDS DEM was 7.13, $17.59 \mathrm{~m}$ for the AU DEM 3 pre-pc_align and $89.30 \mathrm{~m}$ for AU DEM 3 post$p c \_a l i g n . P c \_a l i g n$ using the MOLA PEDR dramatically increased the RMSE (in reference to the MOLA PEDR) of the AU DEM to $143.58 \mathrm{~m}$.

\section{A3 AU DEM 4}

Site 4 is a glacier-like form in Protonilus Mensae (Figs. 1 and 3 in the main text). A frequent HiRISE target, the glacier-like form is a striking example of the landform (Souness et al., 2012). AU DEM 4 was generated using the HiRISE image ]pair ESP_052535_2225 (left) and ESP_052324_2225 (right). The site 4 PDS DEM is DTEEC_019358_2225_018857_2225_U01 (https://bit.ly/ 2VSdUFT, last access: 10 February 2019), and the HRSC DEM is h1523_0000.

Site 4 is the only site at which $p c \_a l i g n$ did not alter the AU DEM dramatically. Compared to the PDS DEM, the RMSE of AU DEM 4 pre-pc_align was 23.93 and $23.07 \mathrm{~m}$ after $p c \_a l i g n$. Removing slopes $>10^{\circ}$ lowered the RMSE of AU DEM 4 pre-pc_align to $9.75 \mathrm{~m}$, but for the post$p c \_a l i g n$ AU DEM, RMSE increased by $0.35 \mathrm{~m}$. Although the shift was subtle (Fig. A4), Fig. A3c indicates the AU DEM is offset by a constant $100 \mathrm{~m}$ in a northwest direction prior to $p c \_a l i g n$. However, $p c \_a l i g n$ appears to have rotated AU DEM 4 clockwise (Fig. A3d and e) with the centre of this rotation in the upper northwest corner. Low displacement in the upper-left-hand corner (where the steepest slopes are located), removed only pixels which were in goodagreement between each DEM. In contrast, the constant offset of the pre-pc_align AU DEM meant that with steep slopes removed, only the low-gradient surfaces remained. Because elevation remains approximately constant across large areas where slope is low, the RMSE of the two DEMs decreased by $>10 \mathrm{~m}$.

Between each of the AU DEMs and the PDS DEM, the RMSE against the HRSC DEM was very similar. The RMSE of the AU DEM was $51.57 \mathrm{~m}$ pre- and $51.57 \mathrm{~m}$ post-pc_align. 
The RMSE of the PDS DEM was $52.74 \mathrm{~m}$ against the HRSC DEM. In contrast, the RMSE of the PDS DEM in reference to the MOLA PEDR was $10.82 \mathrm{~m}$, whereas the RMSE of the AU DEM was $29.43 \mathrm{~m}$ pre- and $26.36 \mathrm{~m}$ post-pc_align.

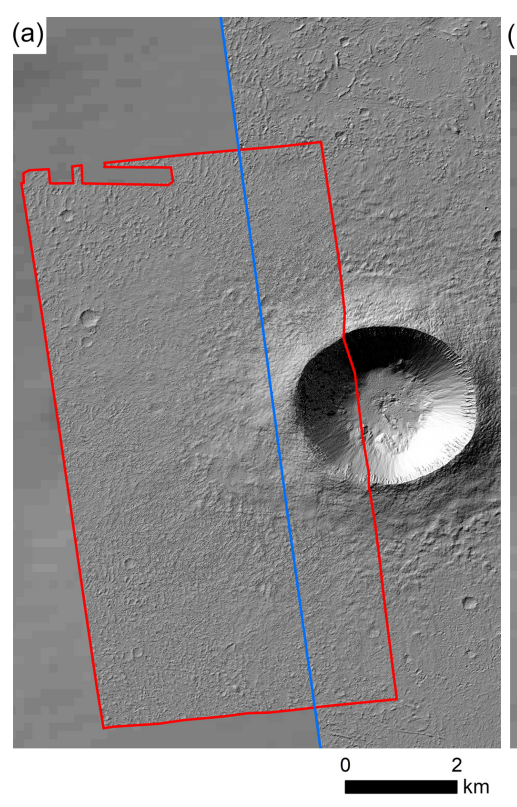

(c)

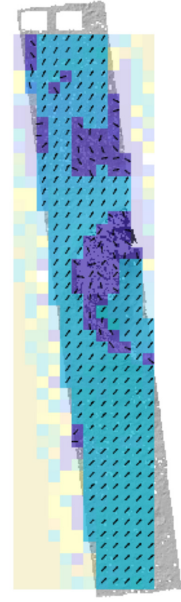

(d)
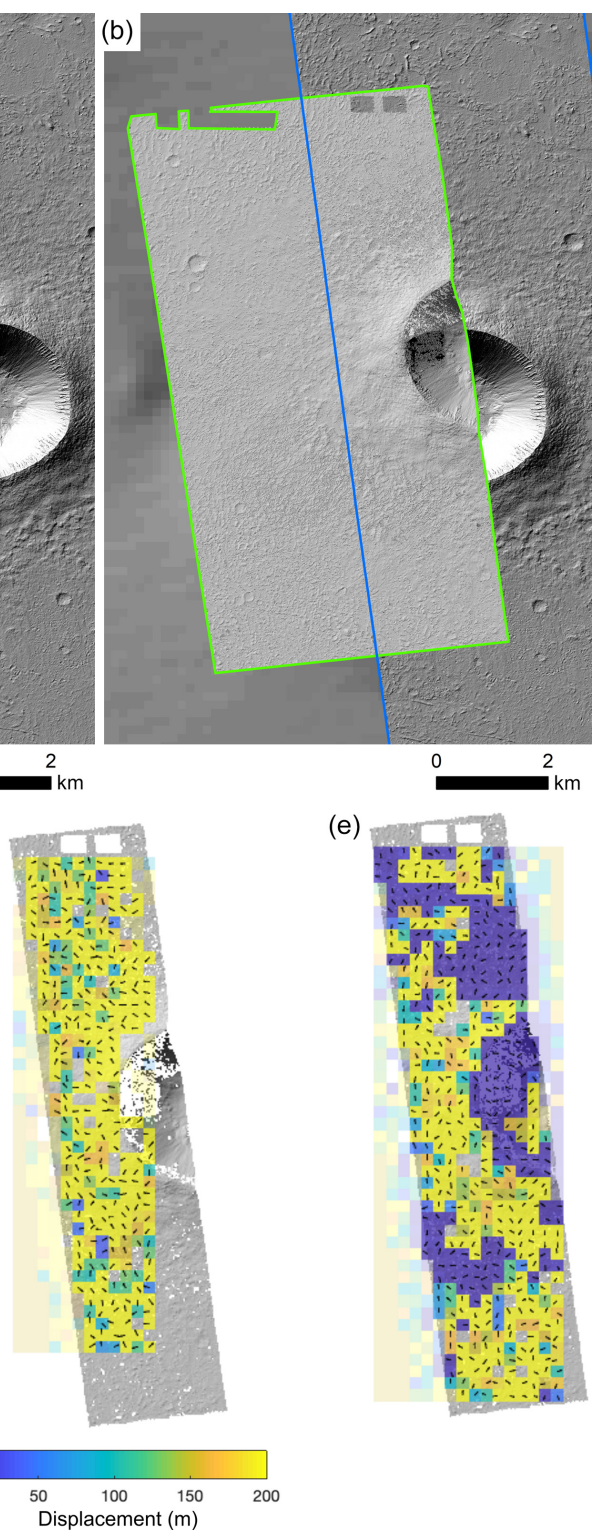

(e)

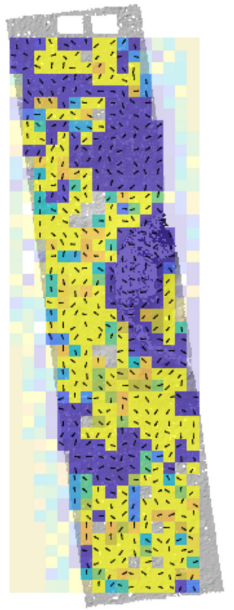

Figure A1. Alignment of DEMs for site 1 including the AU DEM produced using the pipeline presented, the PDS DEM (DTEEC_002118_1510_003608_1510_A01: https://bit.ly/2tSD8Ig, last access: 9 February 2019), and the HRSC DEM (h2538_0000_da4). (a-b) Hillshaded PDS DEM (blue box), the AU DEM 3 pre-pc_align (red box), and AU DEM 3 post-pc_align (green box). Background is the hillshaded HRSC DEM. Note the large misalignment between AU and PDS DEMs after pc_align. (c) Displacement between the ASP (pre-pc_align) DEM and the PDS DEM. Little displacement indicates strong agreement. (d) Displacement measured between the two AU DEMs. Overlap between the two DEMs is limited to flat-featureless terrain, making pixel matching between the two DEMs difficult. (e) Displacement between the ASP (post-pc_align) DEM and the PDS DEM. As with (d), low-texture terrain limits the efficacy of displacement tracking. Panels (c-e) created using the ImGRAFT feature tracking algorithm (Messerli and Grinsted, 2015). 


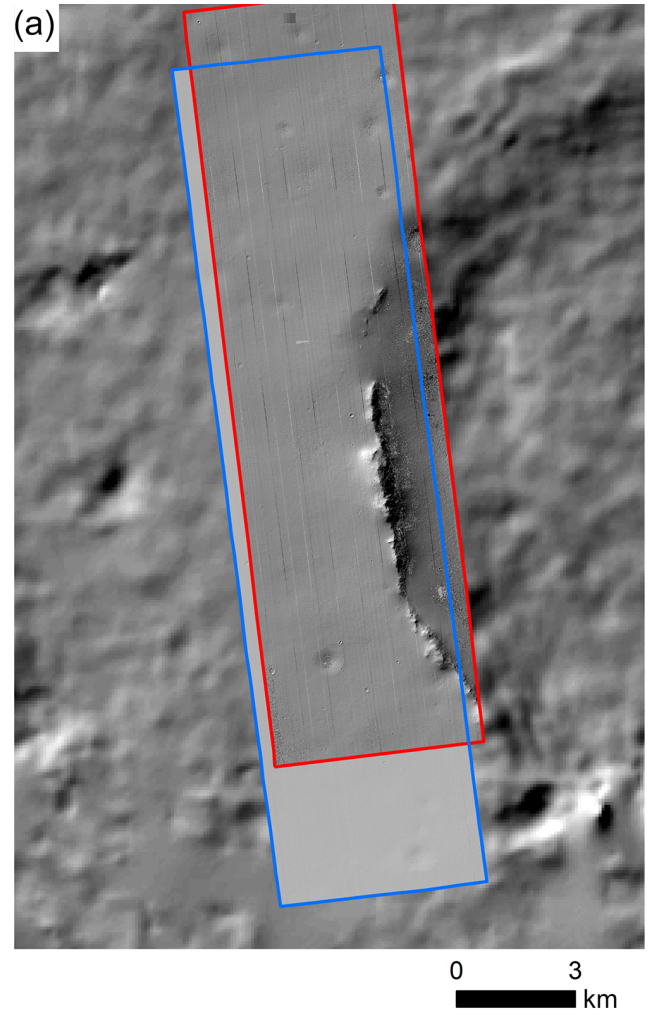

(c)

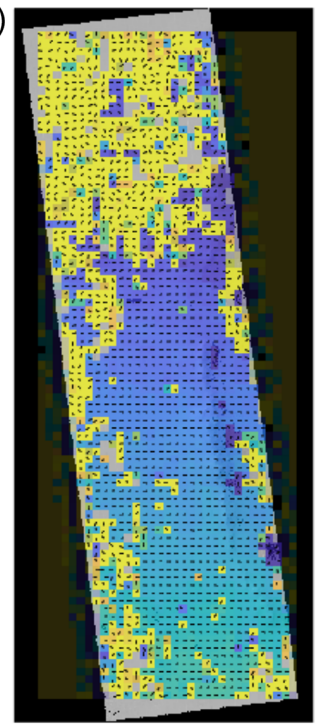

(d)

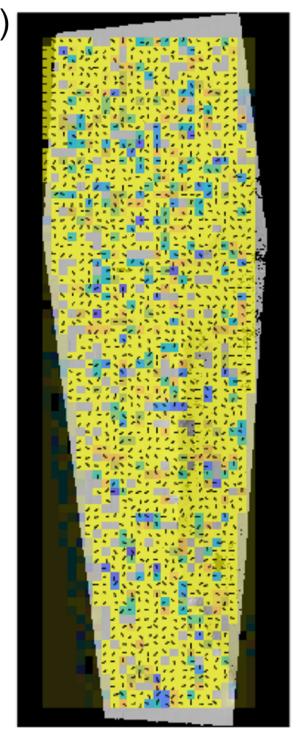

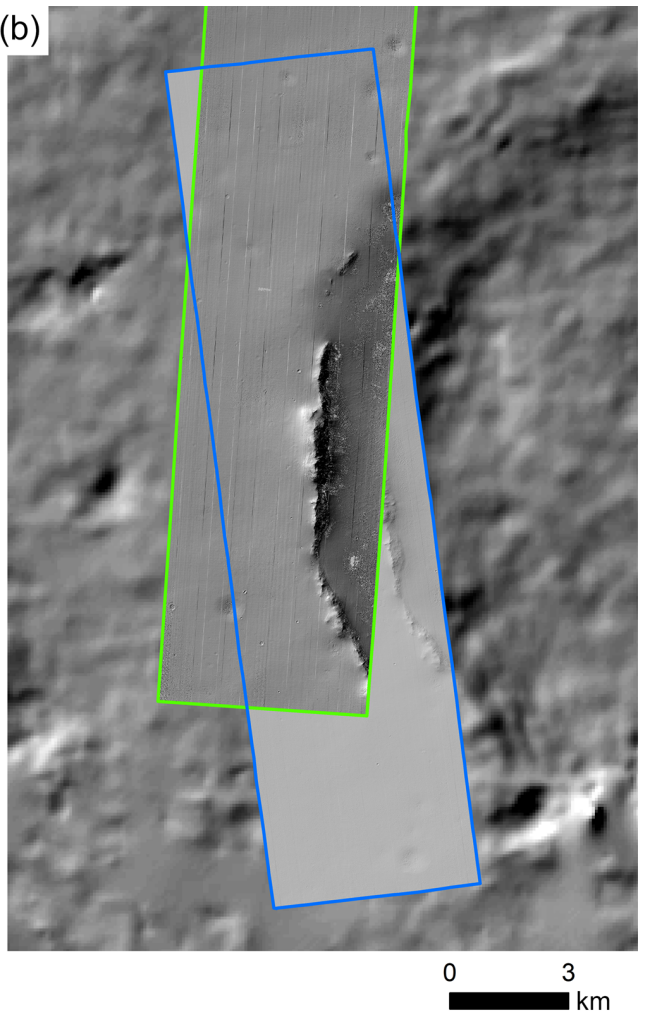

(e)

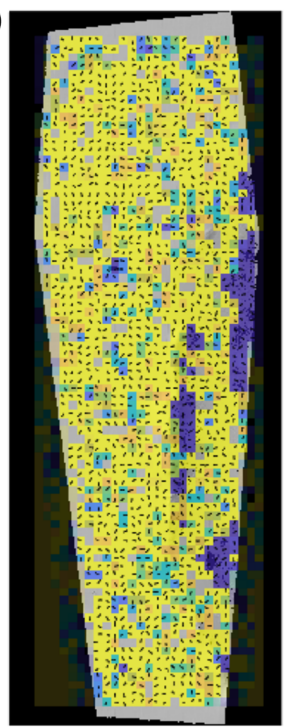

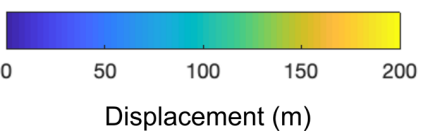

Figure A2. Site 3 DEM alignment between AU DEM 3, the PDS DEM (DTEEC_018701_1775_018846_1775_U01: https://bit.ly/2TrxFXX, last access: 9 February 2019), and the HRSC DEM (h3198_0001). (a-b) Hillshaded PDS DEM (blue box), the AU DEM 3 pre-pc_align (red box), and AU DEM 3 post-pc_align (green box). Background is the hillshaded HRSC DEM. Note the longitudinal artefacts associated with HiRISE bands. (c) Displacement between the AU (pre-pc_align) DEM and the PDS DEM. Low displacement around the crater rim indicates strong agreement. Elsewhere, the low-texture terrain hampers pixel matching. (d) Displacement measured between the two AU DEMs. Overlap is limited to flat-featureless terrain, making pixel matching between the two DEMs difficult. (e) Displacement between the ASP (post-pc_align) DEM and the PDS DEM. As with (d), low-texture terrain limits the efficacy of displacement tracking. Panels (c-e) created using the ImGRAFT feature tracking algorithm (Messerli and Grinsted, 2015). 

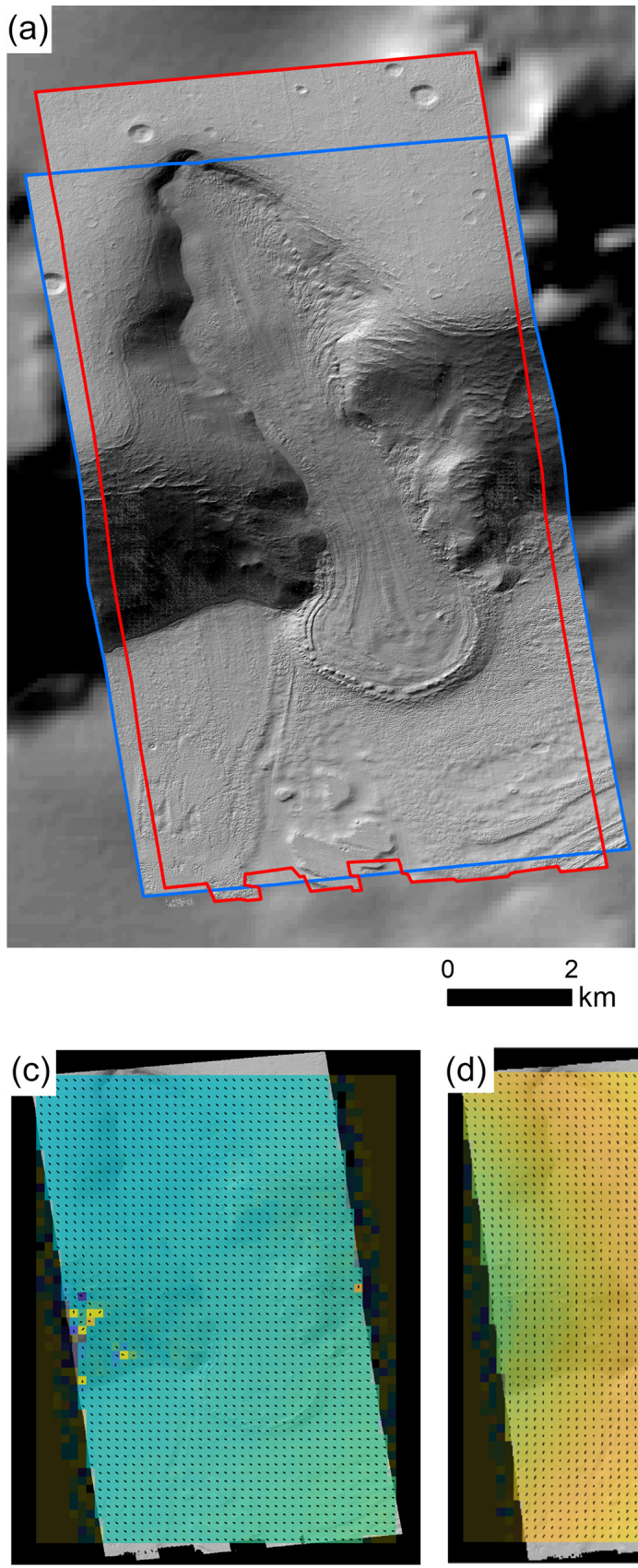

(b)

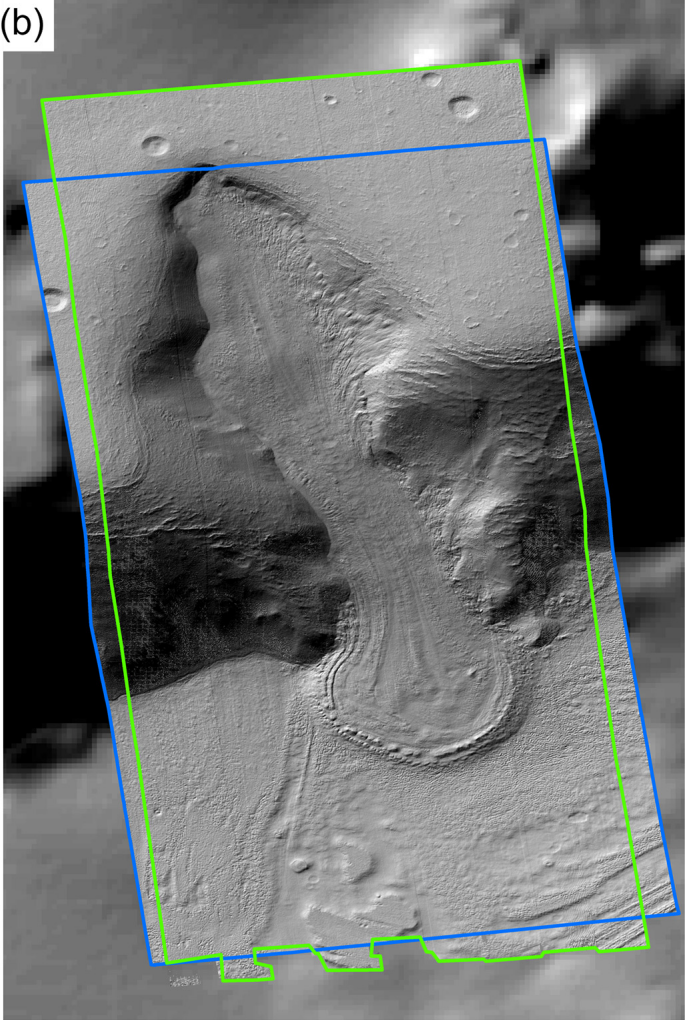

0

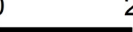

$\mathrm{km}$
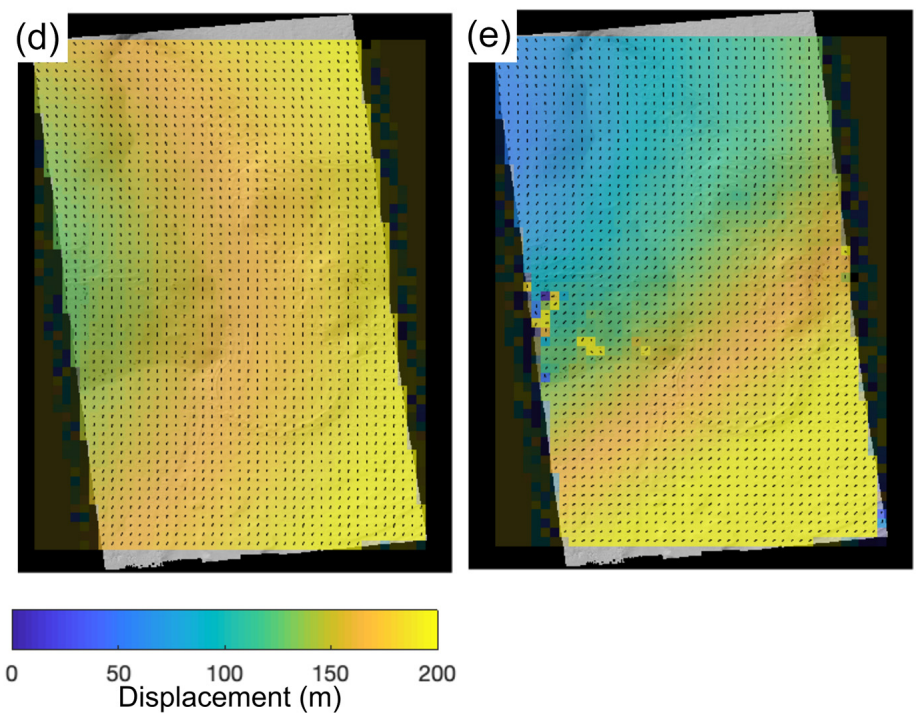

Figure A3. DEM alignment at site 4 between AU DEM 4, the PDS DEM (DTEEC_019358_2225_018857_2225_U01: https://bit.ly/ 2VSdUFT, last access: 9 February 2019), and the HRSC DEM (h1523_0000). (a-b) Hillshaded PDS DEM (blue box), the AU DEM 4 pre-pc_align (red box), and AU DEM 4 post-pc_align (green box). Background is the hillshaded HRSC DEM. (c) Displacement vector field between AU DEM 4 pre-pc_align and the PDS DEM. Displacement is a consistent magnitude and orientated in a northwest direction. (d) Displacement vector field between the two AU DEMs. Displacement appears consistent, but with a lower-magnitude area in the northwestern portion of the vector field, suggesting the misalignment was primarily rotational between the two AU DEMs. (e) Displacement vector field between AU DEM 4 post-pc_align and the PDS DEM. Vector field indicates displacement with a strong rotational component. Note the low displacement in the northwestern corner, where the steepest slopes are also located. Panels (c-e) created using the ImGRAFT feature tracking algorithm (Messerli and Grinsted, 2015). 


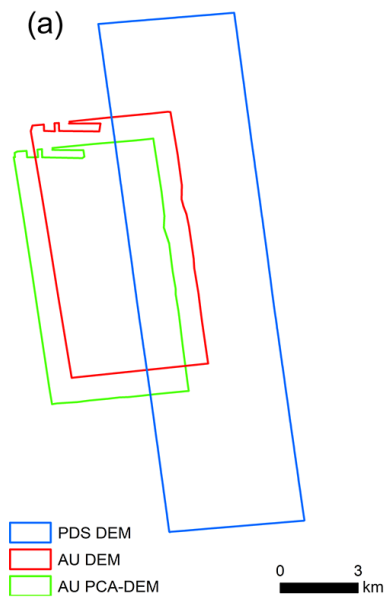

(b)

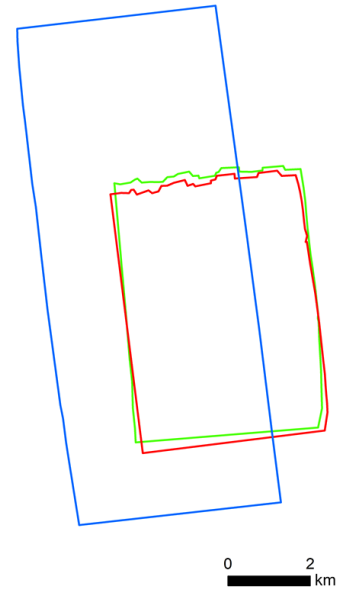

(c)

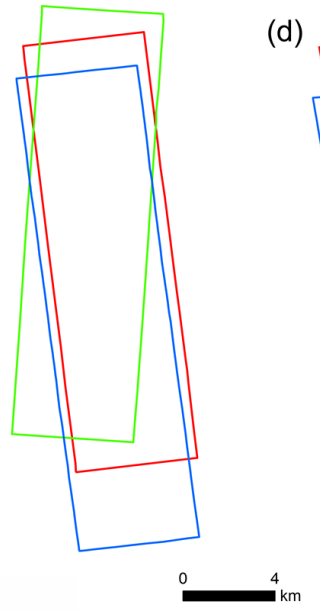

(d)

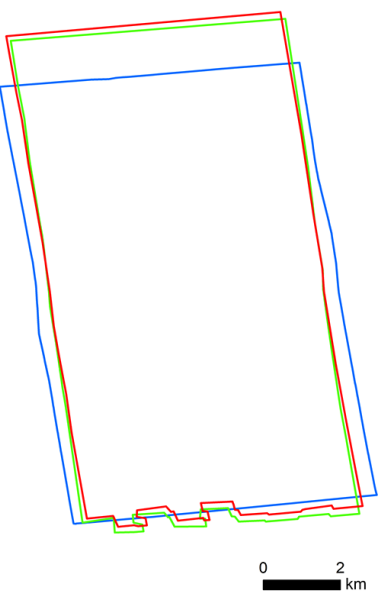

Figure A4. Footprints generated from each HiRISE DEM (AU and PDS) for each of the four sites. Note the large shifts after $p c \_a l i g n$ with the AU DEMs for sites 1 (a) and 3 (b). In each of these cases, $p c \_$align dramatically increased the RMSE of AU DEMs against the reference DEMs. 
Author contributions. AJH performed all method work and produced the paper. $\mathrm{AJH}, \mathrm{TH}$, and $\mathrm{BH}$ designed the study and analysed the results. All authors discussed the results and commented on the writing.

Competing interests. The authors declare that they have no conflict of interest.

Acknowledgements. Thanks are extended to Samuel Doyle for his help teaching Adam J. Hepburn how to use LaTeX. Adam J. Hepburn would also like to thank Jayne Kamintzis for proofreading the paper. We thank NASA/JPL/University of Arizona for making available all of the data used in this study.

Review statement. This paper was edited by Marina Di'azMichelena and reviewed by three anonymous referees.

\section{References}

Adler, J. B., Bell, J. F., Fawdon, P., Davis, J., Warner, N. H., Sefton-nash, E., and Harrison, T. N.: Hypotheses for the origin of the Hypanis fan-shaped deposit at the edge of the Chryse escarpment, Mars: Is it a delta?, Icarus, 319, 885-908, https://doi.org/10.1016/j.icarus.2018.05.021, 2019.

Anderson, J. A.: ISIS Camera Model Design, Abstract 2159, Lunar and Planetary Science XXXIX, 2008.

Anderson, J. A., Sides, S. C., Soltesz, D. L., Sucharski, T. L., and Becker, K. J.: Modernization of the Integrated Software for Imagers and Spectrometers, Abstract 2039, Lunar and Planetary Science XXXV, 2004.

Andrieu, F., Schmidt, F., Douté, S., and Chasse, E.: Ice state evolution during spring in Richardson crater, Mars, Icarus, 315, 158173, https://doi.org/10.1016/j.icarus.2018.06.019, 2018.

Aye, K., Schwamb, M. E., Portyankina, G., Hansen, C. J., Mcmaster, A., Miller, G. R. M., Carstensen, B., Snyder, C., Parrish, M., Lynn, S., Mai, C., Miller, D., Simpson, R. J., Smith, A. M., Observatory, G., and Place, N. A.: Planet Four: Probing springtime winds on Mars by mapping the southern polar $\mathrm{CO} 2$ jet deposits, Icarus, 319, 558-598, https://doi.org/10.1016/j.icarus.2018.08.018, 2019.

Baker, S. and Matthews, I.: Lucas-Kanade 20 Years On: A Unifying Framework, Int. J. Comput. Vis., 56, 221-255, 2004.

Banks, M. E., Fenton, L. K., Bridges, N. T., and Geissler, P. E.: Patterns in Mobility and Modification of Middle- and High-Latitude Southern Hemisphere Dunes on Mars, J. Geophys. Res., 123, 3205-3219, https://doi.org/10.1029/2018JE005747, 2018.

Berman, D. C., Balme, M. R., Michalski, J. R., Clark, S. C., and Joseph, E. C. S.: High-resolution investigations of Transverse Aeolian Ridges on Mars, Icarus, 312, 247-266, https://doi.org/10.1016/j.icarus.2018.05.003, 2018.

Beyer, R. A., Alexandrov, O., and McMichael, S.: The Ames Stereo Pipeline: NASA's Open Source Software for Deriving and Processing Terrain Data, Earth Space Sci., 5, 537-548, https://doi.org/10.1029/2018EA000409, 2018.
Chojnacki, M., Johnson, J. R., Moersch, J. E., Fenton, L. K., Michaels, T. I., and Bell, J. F.: Persistent aeolian activity at Endeavour crater, Meridiani Planum, Mars; new observations from orbit and the surface, Icarus, 251, 275-290, https://doi.org/10.1016/j.icarus.2014.04.044, 2014.

Chojnacki, M., Urso, A., Fenton, L. K., and Michaels, T. I.: Aeolian dune sediment flux heterogeneity in Meridiani Planum, Mars, Aeolian Res., 26, 73-88, https://doi.org/10.1016/j.aeolia.2016.07.004, 2017.

Chuang, F. C., Crown, D. A., and Tornabene, L. L.: Zumba crater, Daedalia Planum, Mars: Geologic investigation of a young, rayed impact crater and its secondary field, Icarus, 269, 75-90, https://doi.org/10.1016/j.icarus.2016.01.005, 2016.

Conway, S. J. and Balme, M. R.: Decameter thick remnant glacial ice deposits on Mars, Geophys. Res. Lett., 41, 5402-5409, https://doi.org/10.1002/2014GL060314, 2014.

Davis, J., Grindrod, P., Fawdon, P., Williams, R., Gupta, S., and Balme, M. R.: Episodic and Declining Fluvial Processes in Southwest Melas Chasma, Valles Marineris, Mars, J. Geophys. Res.-Planet., 123, 2527-2549, https://doi.org/10.1029/2018JE005710, 2018.

Day, M. and Kocurek, G.: Observations of an aeolian landscape: From surface to orbit in Gale Crater, Icarus, 280, 37-71, https://doi.org/10.1016/j.icarus.2015.09.042, 2016.

Fenton, L. K. and Herkenhoff, K. E.: Topography and Stratigraphy of the Northern Martian Polar Layered Deposits Using Photoclinometry, Stereogrammetry, and MOLA Altimetry, Icarus, 148, 433-443, https://doi.org/10.1006/icar.2000.6459, 2000.

Frey, H., Sakimoto, S. E., and Roark, J.: The MOLA topographic signature at the crustal dichotomy boundary zone on Mars, Geophys. Res. Lett., 25, 4409-4412, https://doi.org/10.1029/1998GL900095, 1998.

Golombek, M., Grant, J., Kipp, D., Vasavada, A., Kirk, R., Fergason, R., Bellutta, P., Calef, F., Larsen, K., Katayama, Y., Huertas, A., Beyer, R., Chen, A., Parker, T., Pollard, B., Lee, S., Sun, Y., Hoover, R., Sladek, H., Grotzinger, J., Welch, R., Noe Dobrea, E., Michalski, J., and Watkins, M.: Selection of the Mars Science Laboratory Landing Site, Space Sci. Rev., 170, 641-737, https://doi.org/10.1007/s11214-012-9916-y, 2012.

Golombek, M., Kipp, D., Warner, N., Daubar, I., Fergason, R., Kirk, R., Beyer, R., Huertas, A., Piqueux, S., Putzig, N., Campbell, B., Morgan, G., Charalambous, C., Pike, W., Gwinner, K., Calef, F., Kass, D., Mischna, M., Ashley, J., Bloom, C., Wigton, N., Hare, T., Schwarts, C., Gengl, H., Redmond, L., Trautman, M., Sweeney, J., Grima, C., Smith, I., Sklyanskiy, E., Lisano, M., Benardini, J., Smrekar, S., Lognonné, P., and Banerdt, W.: Selection of the InSight Landing Site, Space Sci. Rev., 211, 5-95, https://doi.org/10.1007/s11214-016-0321-9, 2017.

Grant, J. A., Golombek, M. P., Wilson, S. A., Farley, K. A., Williford, K. H., and Chen, A.: The science process for selecting the landing site for the 2020 Mars rover, Planet. Space Sci., 164, 106-126, https://doi.org/10.1016/j.pss.2018.07.001, 2018.

Gwinner, K., Jaumann, R., Hauber, E., Hoffmann, H., Heipke, C., Oberst, J., Hiesinger, H., Hoekzema, N. M., Kersten, E., Loizeau, D., Matz, K., Mcguire, P. C., Mertens, V., Michael, G., Pasewaldt, A., Pinet, P., Preusker, F., Reiss, D., Roatsch, T., Schmidt, R., Scholten, F., Spiegel, M., Stesky, R., Tirsch, D., Gasselt, S. V., Walter, S., Wählisch, M., and Willner, K.: The High Resolution Stereo Camera (HRSC) of Mars 
Express and its approach to science analysis and mapping for Mars and its satellites, Planet. Space Sci., 126, 93-138, https://doi.org/10.1016/j.pss.2016.02.014, 2016.

Hamilton, C. W., Mouginis-Mark, P., Sori, M., Scheidt, S. P., and Bramson, A. M.: Episodes of Aqueous Flooding and Effusive Volcanism Associated With Hrad Vallis, Mars, J. Geophys. Res.Planet., 123, 1484-1510, https://doi.org/10.1029/2018JE005543, 2018.

Hubbard, B., Milliken, R. E., Kargel, J. S., Limaye, A., and Souness, C.: Geomorphological characterisation and interpretation of a mid-latitude glacier-like form: Hellas Planitia, Mars, Icarus, 211, 330-346, https://doi.org/10.1016/j.icarus.2010.10.021, 2011.

Jaumann, R., Neukum, G., Behnke, T., Duxbury, T. C., Eichentopf, K., Flohrer, J., Van Gasselt, S., Giese, B., Gwinner, K., Hauber, E., Hoffmann, H., Hoffmeister, A., Köhler, U., Matz, K. D., McCord, T. B., Metens, V., Oberst, J., Pischel, R., Reiss, D., Ress, E., Roatsch, T., Saiger, P., Scholten, F., Schwarz, G., Stephan, K., Wählisch, M., and the HRSC Co-Investigator Team: The highresolution stereo camera (HRSC) experiment on Mars Express: Instrument aspects and experiment conduct from interplanetary cruise through the nominal mission, Planet. Space Sci., 55, 928952, https://doi.org/10.1016/j.pss.2006.12.003, 2007.

Malin, M. C., Bell III, J. F., Cantor, B. A., Caplinger, M. A., Calvin, W. M., Clancy, R. T., Edgett, K. S., Edwards, L., Haberle, R. M., James, P. B., Lee, S. W., Ravine, M. A., Thomas, P. C., and Wolff, M. J.: Context Camera Investigation on board the Mars Reconnaissance Orbiter, J. Geophys. Res., 112, 1-25, https://doi.org/10.1029/2006JE002808, 2007.

Mayer, D. P. and Kite, E. S.: An Intergrated Workflow for Producing Digital Terrain Models of Mars from CTX and HiRISE Stereo Data Using the NASA Ames Stereo Pipeline, Abstract 1241, 47th Lunar and Planetary Science Conference, 2016.

McEwen, A. S.,Eliason, E. M., Bergstrom, J. W., Bridges, N. T., Hansen, C. J., Delamere, W. A., Grant, J. A., Gulick, V. C., Herkenhoff, K. E., Keszthelyi, L., Kirk, R. L., Mellon, M. T., Squyres, S. W., Thomas, N., and Weitz, C. M.: Mars Reconnaissance Orbiter's High Resolution Imaging Science Experiment (HiRISE), J. Geophys. Res., 112, E05S02, https://doi.org/10.1029/2005JE002605, 2007.

Mège, D. and Masson, P.: A plume tectonics model for the Tharsis province, Mars, Planet. Space Sci., 44, 186-189, 1996.

Messerli, A. and Grinsted, A.: Image georectification and feature tracking toolbox: ImGRAFT, Geosci. Instrum. Method. Data Syst., 4, 23-34, https://doi.org/10.5194/gi-4-23-2015, 2015.

Mittlefehldt, D. W., Gellert, R., Ming, D. W., Yen, A. S., Jolliff, B. L., Arvidson, R. E., Farrand, W. H., Herkenhoff, K. E., Bell, I. F., Cohen, B., Klingelhöfer, G., Schrader, C., and Rice, J.: Diverse Lithologies and Alteration Events on the Rim of Noachian-Aged Endeavour Crater, Meridiani Planum, Mars: In Situ Compositional Evidence, J. Geophys. Res.-Planets, 123, 1255, https://doi.org/10.1002/2017JE005474, 2018.

Moratto, S. Z. M., Broxton, M. J., Beyer, R. A., Lundy, M., and Husmann, K.: Ames Stereo Pipeline, NASA's Open Source Automated Stereogrammetry, Abtstract 2364, 41st Lunar and Planetary Science Conference, 1-2, 2010.

Nefian, A. V., Husmann, K., Broxton, M., To, V., Lundy, M., and Hancher, M. D.: A Baysian formulation for sub-pixel refinement in stereo orbital imagery, 2009 16th IEEE Interna- tional Conference on Image Processing (ICIP), 2361-2364, https://doi.org/10.1109/ICIP.2009.5413749, 2009.

Noh, M.-J. and Howat, I. M.: The Surface Extraction from TIN based Search-space Minimization (SETSM) algorithm, ISPRS J. Photogramm. Remote Sens., 129, 55-76, https://doi.org/10.1016/j.isprsjprs.2017.04.019, 2017.

Schaefer, E. I., Mcewen, A. S., and Sutton, S. S.: A case study of recurring slope lineae (RSL) at Tivat crater: Implications for RSL origins, Icarus, 317, 621-648, https://doi.org/10.1016/j.icarus.2018.07.014, 2019.

Sharp, R. P.: Mars : Fretted and Chaotic Terrains possible genesis of these two terrain types, J. Geophys. Res., 78, 4073-4083, 1973.

Shin, D. and Muller, J.-P.: Progressively weighted affine adaptive correlation matching for quasi-dense 3D reconstruction, Pattern Recog., 45, 3795-3809, https://doi.org/10.1016/j.patcog.2012.03.023, 2012.

Smith, D. E., Zuber, M. T., Frey, H. V., Garvin, J. B., Head, J. W., Muhleman, D. O., Pettengill, G. H., Phillips, R. J., Solomon, S. C., Zwally, H. J., Banerdt, W. B., Duxbury, T. C., Golombek, M. P., Lemoine, F. G., Neumann, G. A., Rowlands, D. D., Aharonson, O., Ford, P. G., Ivanov, A. B., Johnson, C. L., McGovern, P. J., Abshire, J. B., Afzal, R. S., and Sun, X.: Mars Orbiter Laser Altimeter: Experiment summary after the first year of global mapping of Mars, J. Geophys. Res., 106, 23689, https://doi.org/10.1029/2000JE001364, 2001.

Souness, C., Hubbard, B., Milliken, R. E., and Quincey, D.: An inventory and population-scale analysis of martian glacier-like forms, Icarus, 217, 243-255, https://doi.org/10.1016/j.icarus.2011.10.020, 2012.

Stack, K. M., Edwards, C. S., Grotzinger, J. P., Gupta, S., Sumner, D. Y., Calef, F., Edgar, L., Edgett, K., Fraeman, A., Jacob, S., Le Deit, L., Lewis, K., Rice, M., Rubin, D., Williams, R., and Williford, K.: Comparing orbiter and rover image-based mapping of an ancient sedimentary environment, Aeolis Palus, Gale crater, Mars, Icarus, 280, 3-21, 2016.

Stein, N., Arvidson, R., O’Sullivan, J., Catalano, J., Guinness, E., Politte, D., Gellert, R., and VanBommel, S.: Retrieval of Compositional End-Members From Mars Exploration Rover Opportunity Observations in a Soil-Filled Fracture in Marathon Valley, Endeavour Crater Rim, J. Geophys. Res.-Planet., 123, 278-290, https://doi.org/10.1002/2017JE005339, 2018.

Tao, Y., Muller, J., Sidiropoulos, P., Xiong, S.-t., Putri, A. R. D., Walter, S. H. G., Veitch-michaelis, J., and Yershov, V.: Massive stereo-based DTM production for Mars on cloud computers, Planet. Space Sci., 154, 30-58, https://doi.org/10.1016/j.pss.2018.02.012, 2018.

Tirsch, D., Bishop, J. L., Voigt, J. R. C., Tornabene, L. L., Erkeling, G., and Jaumann, R.: Geology of central Libya Montes, Mars: Aqueous alteration history from mineralogical and morphological mapping, Icarus, 314, 12-34, https://doi.org/10.1016/j.icarus.2018.05.006, 2018.

Triggs, B., Mclauchlan, P., Hartley, R., and Fitzgibbon, A.: Bundle Adjustment - A Modern Synthesis, International Workshop on Vision Algorithms, Sep 2000, Corfu, Greece, 298-372, 2000.

Voigt, J. R. C. and Hamilton, C. W.: Investigating the volcanic versus aqueous origin of the surficial deposits in Eastern Elysium Planitia, Mars, Icarus, 309, 389-410, https://doi.org/10.1016/j.icarus.2018.03.009, 2018. 
Weitz, C., Bishop, J. L., Thollot, P., Mangold, N., and Roach, L. H.: Diverse Mineralogies in Two Troughs of Noctis Labyrinthus, Mars, Geology, 39, 899-902, https://doi.org/10.1130/G32045.1, 2011.
Wilson, S., Grant, J., Howard, A., and Buczkowski, D. The Nature and Origin of Deposits in Uzboi Vallis on Mars, J. Geophys. Res.-Planet., 123, 1842-1862, https://doi.org/10.1029/2017JE005508, 2018. 